SOCIAL INCLUSION WITHIN COMMUNITY YOUTH PROGRAMMING:

\title{
AN EXPLORATION OF THE EXPERIENCES OF FIRST AND SECOND GENERATION YOUTH WITHIN MAINSTREAM, MULTICULTURAL AND ETHNO-SPECIFIC ORGANIZATIONS
}

\author{
by \\ Marleah Beth Eriksson Graff, BA, University of Winnipeg, 2011 \\ A Major Research Paper \\ presented to Ryerson University \\ in partial fulfillment of the requirements for the degree of \\ Master of Arts \\ in the Program of \\ Immigration and Settlement Studies
}

Toronto, Ontario, Canada, 2015

CMarleah Graff 2015 
AUTHOR'S DECLARATION FOR ELECTRONIC SUBMISSION OF A MAJOR RESEARCH PAPER (MRP)

I hereby declare that I am the sole author of this Major Research Paper. This is a true copy of the MRP, including any required final revisions, as accepted by my examiners. I authorize Ryerson University to lend this MRP to other institutions or individuals for the purpose of scholarly research. I further authorize Ryerson University to reproduce this MRP by photocopying or by other means, in total or in part, at the request of other institutions or individuals for the purpose of scholarly research. I understand that my MRP may be made electronically available to the public. 
Social Inclusion within Community Youth Programming:

An Exploration of the Experiences of First and Second Generation Youth within Mainstream, Multicultural and Ethno-specific Organizations

\author{
Marleah Beth Eriksson Graff \\ Master of Arts 2015 \\ Immigration and Settlement Studies \\ Ryerson University
}

\begin{abstract}
This study explores the experiences of first and second generation immigrant youth within community youth programming in mainstream, multicultural and ethno-specific organizations. Through interviews with nine youth and three youth program staff this study reveals how youth view the community based programming they attend as well as how their experiences reflect social inclusion or exclusion. Young people's positive experiences are that youth programs are spaces that generate positive feelings, contribute to growth, assist in developing meaningful relationships and connect youth to their community. However, youth also disclose experiences which negatively impact their inclusion in youth programming. Using a lens of social inclusion, this study demonstrates the central role of community youth programming in creating socially inclusive or exclusive environments. These young people's recommendations for change provide solutions for making community youth programs more inclusive.
\end{abstract}

Key words: first generation youth; second generation youth; community youth programming; social inclusion; social exclusion; immigrants 


\section{ACKNOWLEDGEMENTS}

I would like to express my gratitude to everyone who supported and coached me through my MRP ethics, interview, writing and editing processes.

I would like to especially thank my supervisor, Vappu Tyyskä, for her never-ending patience throughout this experience as well as her advice and editing support. Thank-you as well to my second reader, Kiaras Gharabaghi for his valuable feedback during my oral review.

Many thanks to my family who read my MRP drafts, assisted with editing and encouraged me to continue writing when it was challenging.

Lastly I would like to thank Biya Joba for being my true companion throughout this process, celebrating with me my achievements and encouraging me through my struggles. I would not have been able to accomplish this research paper without his love and support. 


\section{DEDICATION}

This Major Research Paper is dedicated to the youth and staff of the three organizations that participated in this study. Thank you for your honest and enthusiastic involvement. 


\section{TABLE OF CONTENTS}

PAGE

ABSTRACT---

ACKNOWLEDGEMENTS-----_-_-

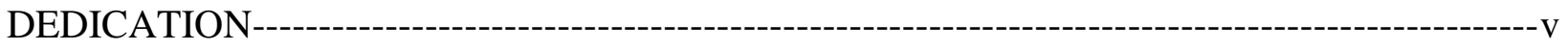

INTRODUCTION---1

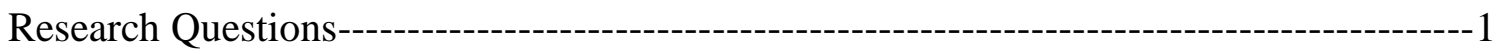

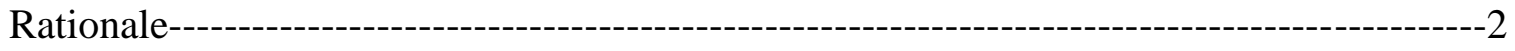

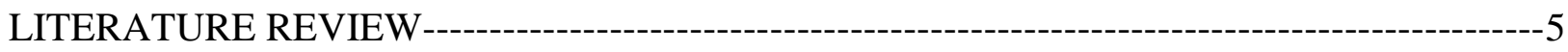

Positive and Negative Experiences of First and Second Generation Immigrant Youth----5

Negative Experiences of First and Second Generation Youth within Settlement,

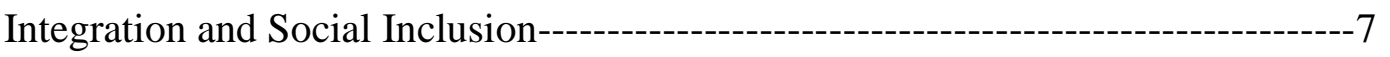

Positive Experiences of First and Second Generation Youth within Settlement,

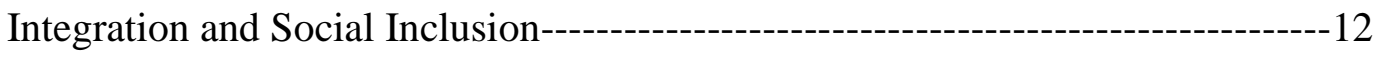

Similarities and Differences between Multicultural, Mainstream and Ethno-specific

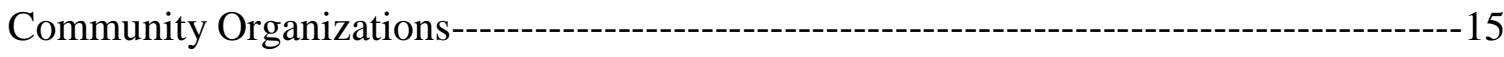

Characteristics of Multicultural Organizations-------------------------------------16

Characteristics of Mainstream Organizations------------------------------------------17 
Characteristics of Ethno-specific Organizations----------------------------------------18

Relationship between Community Programming and Immigrant Youth---------------------19

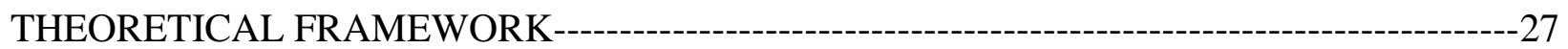

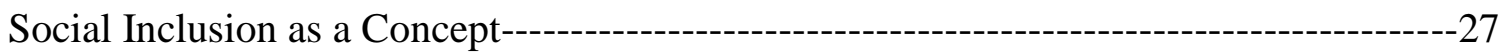

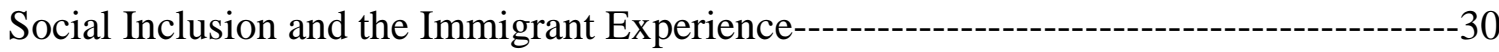

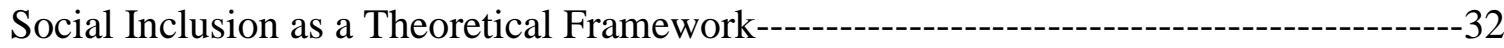

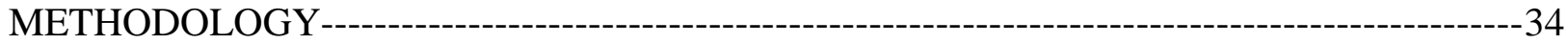

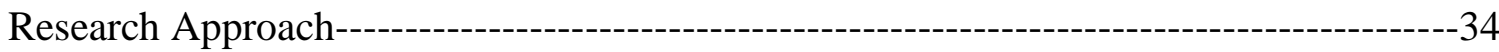

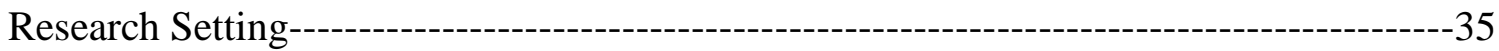

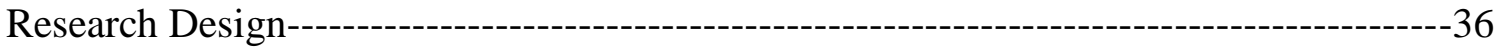

Research Participant Recruitment------------------------------------------------------------------37

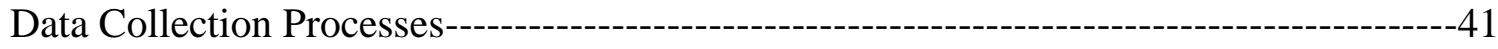

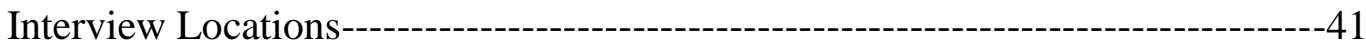

Interview Processes---------------------------------------------------------------------------42

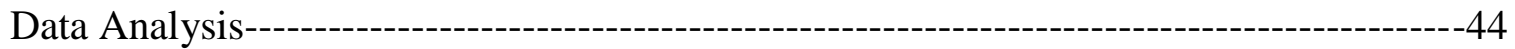

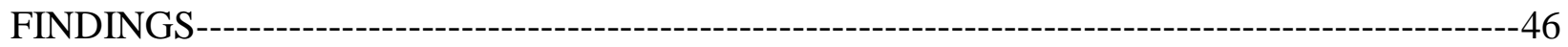

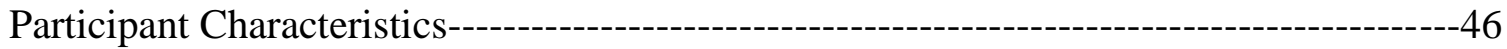




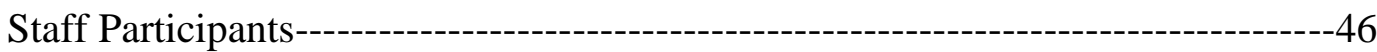

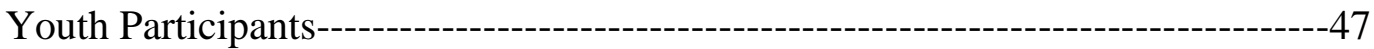

Organizational Profiles----_-

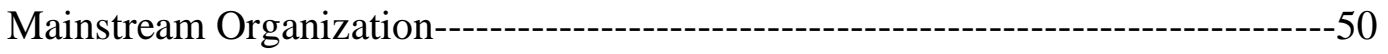

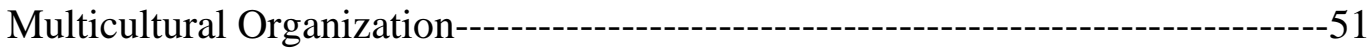

Ethno-specific Organization--------------------------------------------------------52

Young People's Immigration Stories---o-_-

Experiences of First and Second Immigrant Youth in Mainstream, Multicultural and

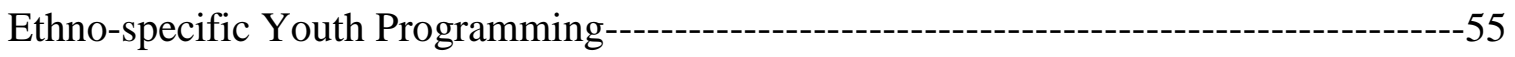

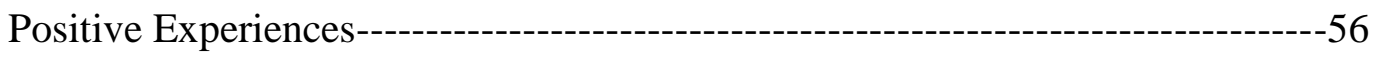

Positive Feelings of 'Happiness', 'Belonging' and 'Pride'-----------------56

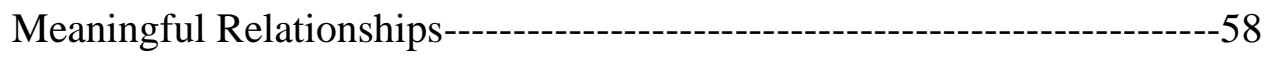

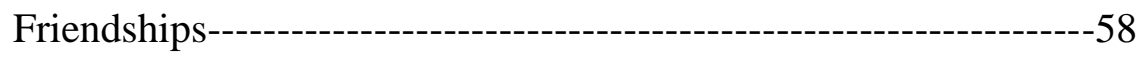

Youth-staff Relationships--------------------------------------------------60

Program-youth Relationship-----------------------------------------61

Connecting with Community------------------------------------------------62

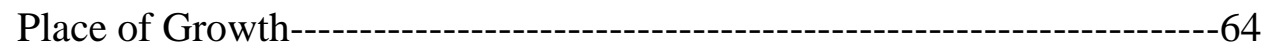

Adaptation to Canadian Life-----------------------------------------64 


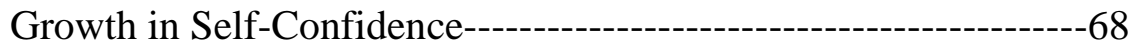

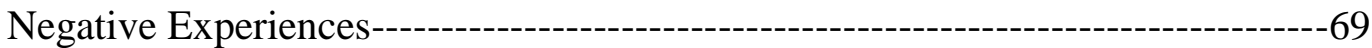

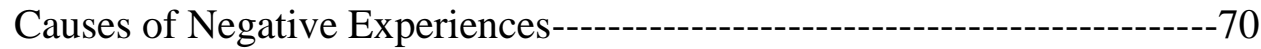

Internal Causes of Negative Experiences------------------------------70

External Causes of Negative Experiences------------------------------71

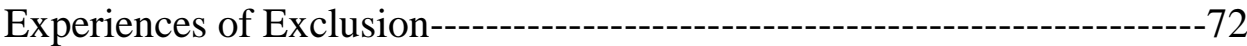

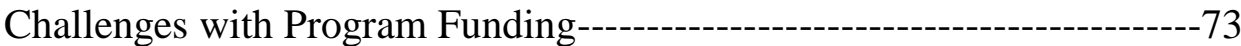

Comparisons on Cultural Experiences of Youth in Mainstream, Multicultural and Ethnospecific Organizations------------------------------------------------------------------------------74

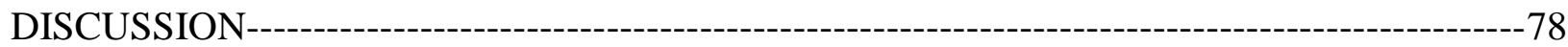

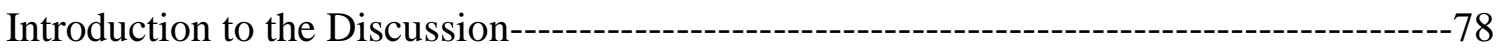

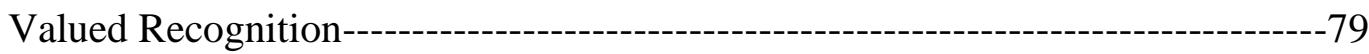

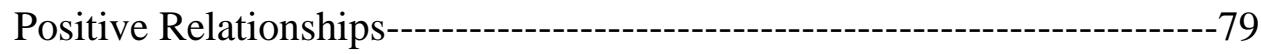

Freedom of Expression and Cultural Association-----------------------------80

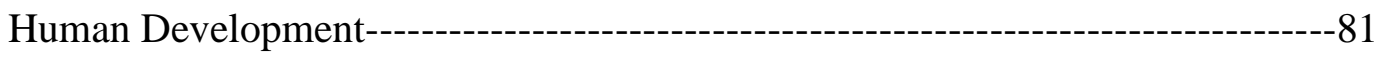

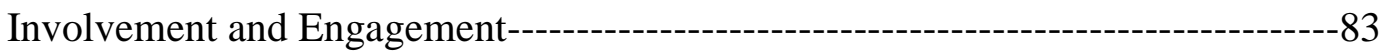




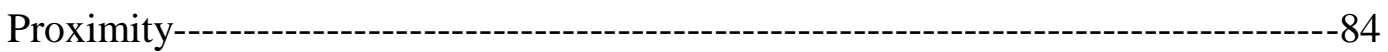

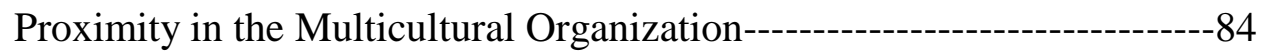

Proximity in the Mainstream Organization------------------------------------84

Proximity in the Ethno-specific Organization--------------------------------85

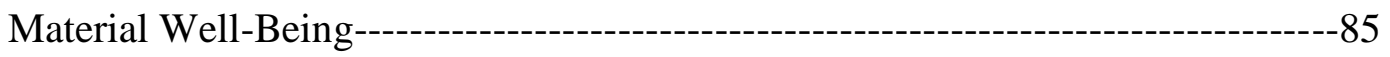

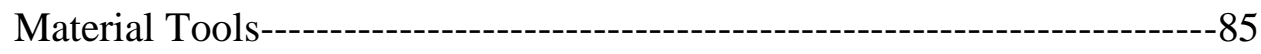

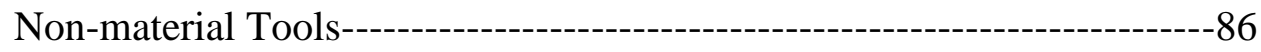

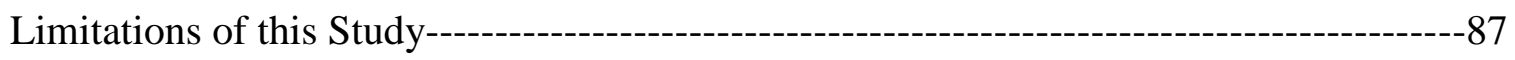

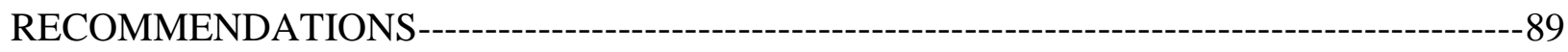

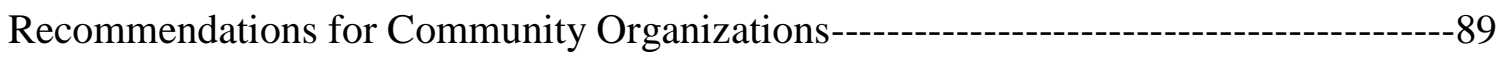

Recommendations for Funding Bodies----------------------------------------------------------91

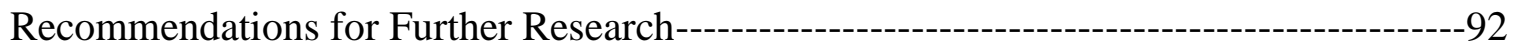

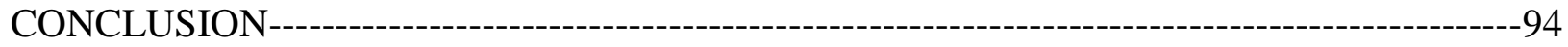

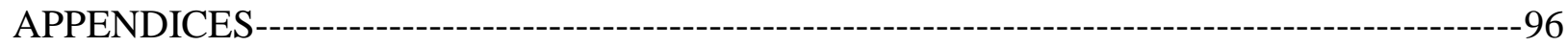

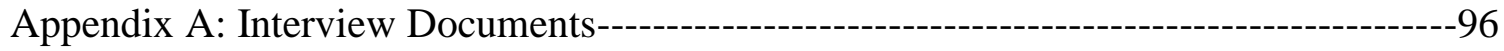

Appendix B: Recruitment Documents-----------------------------------------------------------103

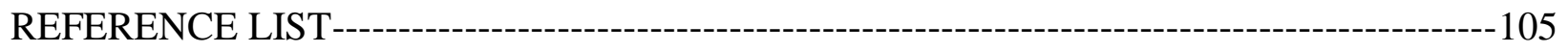




\section{LIST OF TABLES}

PAGE

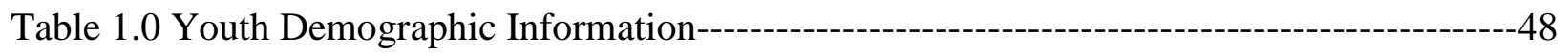




\section{LIST OF APPENDICES}

PAGE

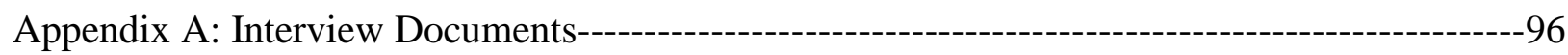

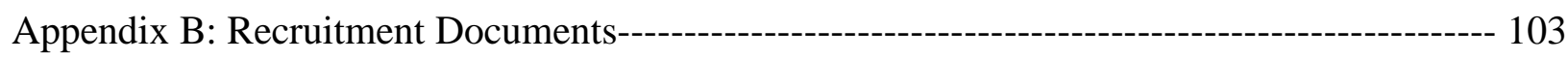




\section{INTRODUCTION}

\section{Research Questions}

First and second generation immigrant youth access a variety of support systems which address their short-term and long-term settlement, integration and social inclusion needs. Informal support systems include friends, family and ethnic or religious communities, while formal support systems include government, non-profit or educational services. A plethora of research focuses on the experiences of first and second generation youth within educational services; however, other support systems are significantly less studied. My own experience as a community worker with various non-profit organizations supports the integral function of community-based services in enabling first and second generation immigrant youth to find the supports they need on their journey toward settlement, integration and finally social inclusion. My perspective comes as a Caucasian, Canadian-born female in her late 20s, leading to the questions: How do first and second generation youth view the community based programming they attend? How do their experiences reflect social inclusion or exclusion?

This study seeks to contribute to research and understanding of newcomer youth community programming through exploring the spectrum of participant experiences within three types of community youth programming: mainstream, multicultural and ethno-specific. First and second generation immigrant youth and youth program staff were interviewed regarding youth's experiences within mainstream, multicultural and ethno-specific community organizations to determine the following: the range of services provided for immigrant youth by community organizations within Toronto; the youth's positive and negative experiences within these three types of organizations and any youth identified gaps within this area of research and programming that should be addressed. The analysis of the narrative clearly shows that 
community organizations are environments of social inclusion for first and second generation youth.

\section{Rationale}

As of 2006, 167,600 newcomers to Canada were aged 15-24 and 223,000 were under the age of 14 (De Villar Nash, 2011). As the population of newcomer children and youth within Canada increases, so will the demand for programming, research and policy that meets their specific needs (Colbert, 2012) therefore, "it is important to understand the success of settlement service initiatives in assisting with their integration" (Thomas, 2012, p. 2). This study views youth as valuable participants in research, as "Listening to children can give....adult researchers an insight into the child's mind that they could not otherwise have" (Ceglowski \& Makovsky, 2012, p. 285). As the researcher, I originally aspired to understand the experiences of only first generation newcomer youth within the three community youth programs; however, once recruitment began, many second generation immigrant youth indicated interest in participating. To include their valuable voices, the study's participation criteria were adapted to include second generation youth. Ngo (2009) remarks that often the voices of newcomer youth are missing from the development of newcomer services and that "Young immigrants are experts in their own socio-cultural realities and know what services are best for them" (Ngo, 2009, p. 95). This study strives to collect the voices and opinions of first and second generation immigrant youth regarding participation in community programming so that youth voices have a greater impact on future development, study and funding of community programming, thus better addressing the needs of first and second generation youth. 
Few studies acknowledge the relationship between community youth programming and first or second generation immigrant youth in Canada. For the purposes of this study, the term community youth programming refers to programming that occurs outside of school hours, is organized by community organizations and can be accessed based on community membership. Community youth programming may include after-school programming, evening or weekend programming, settlement or integration services, recreation, educational, leadership, counselling or employment focused services (Fokkena, 2011; Greenberg, 2013; Ngo, 2009; Rossiter \& Rossiter, 2000). Greenberg (2013) writes that "Although much is known about the beneficial effects of after-school programming for children and youths, the literature focused on immigrant children is sparse" (Greenberg, 2013, p. 101). Due to the increase in numbers of immigrant children and youth accessing evening and weekend youth programming, understanding the extracurricular activities of this population should be a topic to be pursued more actively (Greenberg, 2013).

Working with community organizations, I have witnessed community youth programming fill the needs of newcomer youth from their first year of arrival to after they have become Canadian citizens. In my experience, many programs that, despite lacking resources, facilitate positive connections between newcomer youth and their communities, aid in positive youth identity and support them through their adjustment to life in Canada. Unfortunately, within academia the impact of such organizations and the experiences of newcomer youth within such services is little acknowledged or studied.

Immigration and settlement research primarily focuses on the impact of settlement services on the newcomer community; however, newcomers in Canada also access services from organizations that cater to specific ethnic groups and the mainstream population. Sadiq (2004) 
categorizes the different services accessed by newcomers as mainstream services, multicultural services and ethno-specific services, with each space providing different levels of co-ethnic concentration. According to Sadiq, mainstream services often have a low co-ethnic concentration and run limited culturally-appropriate programming from a euro-centric position. Multicultural services provide a diverse range of settlement services to immigrants from different ethnic backgrounds; however, they may act as a 'one-stop shop', rarely meeting the cultural and linguistic needs of the diverse population they serve (Sadiq, 2004). Lastly, ethno-specific services, often voluntary organizations, provide settlement aid to a specific ethnic or visible minority group, creating social capital in the community, namely "bonding capital" (connections with members of their own ethnic group) and bridging capital (connections with other community organizations and the public)" (Meinhard \& Faridi, 2009, p. 1). By listening to experiences of first and second generation youth participants from all three categories of services, this study will examine whether community youth programming from mainstream, multicultural and ethno-specific services are spaces of social inclusion for first and second generation youth. 


\section{LITERATURE REVIEW}

This literature review seeks to present an academic discussion regarding newcomer youths' experiences and provide an overview of research studying newcomer youths' relationship with community level programming. First, a brief summary of the positive and negative experiences of first and second generation youth will be presented. The second section will explore the similarities and differences between Sadiq's (2004) three categories of newcomer services: mainstream, multicultural and ethno-specific. The final section will review current academic research focusing on the relationship between community programming and immigrant youth.

\section{Positive and Negative Experiences of First and Second Generation Immigrant Youth}

Before discussing the positive and negative experiences of first and second generation immigrant youth, it is important to define the terms first generation newcomer youth and second generation immigrant youth. A good place to start is by looking at the meaning of 'newcomer'. Under the Canadian federal government, a 'newcomer' is often defined as "someone who has been in Canada for three years or less, is not a Canadian citizen, but is someone to whom Canada intends to grant permanent resident status" (Lim, Lo \& Siemiatycki, 2005, p. 5). This definition however excludes certain individuals such as refugee claimants, the undocumented, newcomers who have already gained Canadian citizenship and second generation newcomer youth from participating in services funding by Citizenship and Immigration Canada (Lim et al., 2005).

Many settlement and community organizations have a different interpretation of the term newcomer, as one that encompasses refugee and immigrant populations who are newly arrived in Canada (Quirke, 2011). It is this understanding of the term newcomer that will be applied within this study. All youth who participated in this study have familial and emotional immigrant 
connections; however, for some they are experienced firsthand, while other youth have experienced them through the stories of their families. A first generation newcomer youth is essentially a youth who at birth has no Canadian citizenship, therefore arriving in Canada as a refugee or immigrant (Batalova \& Fix, 2011). As newcomer families settle and form communities, they may give birth to children who are born Canadian but whose family may still be accessing newcomer services and experiencing newcomer related settlement needs (Siahaan, Lee \& Kalist, 2014). Despite being Canadian, these youth are still deeply connected to the newcomer experiences through their family history and day to day experiences and are therefore called second-generation immigrant youth. It is important to note that both first and second generation youth represent a diverse group of youth comprised of different genders, residential and ethnic identities as well as different levels of skills, education, social capital and socioeconomic capabilities (Kobayashi, 2008). Overall, research on the experiences of second generation youth is less developed than research on first generation newcomer youth, however as the first section of this literature review explores there remain several areas in which the two generations may be compared and contrasted in regards to their various positive and negative experiences (Khanlou, 2008).

The term 'youth' is also a term regularly debated, sometimes referring to an actual age bracket (i.e. 15-24) or based on more conceptual defining factors of dependency or maturity (Tyyskä, 2001). The term youth may also apply to individuals sharing certain characteristics. When this study began, I conceptualized youth as individuals aged 12-18 based off of the age requirements for many of the youth programs that I was researching. However, during the recruitment process, it was clear that many individuals older than 18 still participated in youth programming and referred to themselves as 'youth'. Therefore, in this study youth will be 
considered a young person between the ages 12 and 24 who linger between childhood and adulthood.

\section{Negative Experiences of First and Second Generation Youth within Settlement, Integration and Social Inclusion}

First and second generation immigrant youth have many strengths. Those youth who have immigrated to Canada have done so "with enormous potential to make a significant, positive contribution to the future of their adopted country" (Rossiter \& Rossiter, 2009, p. 410). However, academic research is overwhelmingly focused on understanding and responding to the negative experiences of first generation newcomer youth during settlement, integration and social inclusion. Recent studies have examined structural barriers to academic achievement (Grahahm \& Juvonen, 2002; Karlovic, 2004; Ontario Council of Agencies Serving Immigrants, 2009; Phan, 2003), low English/French language proficiency (Karlovic, 2004; Ontario Council of Agencies Serving Immigrants, 2009), employment barriers (Quirke, 2011; Yan, Lauer \& Jhangiani, 2008), cultural conflict (Ontario Council of Agencies Serving Immigrants, 2009; Zhou, 1997), mental health (Beiser, Taa, Fenta-Wube, Pain \& Araya, 2012), intergenerational conflict (Hyman, Vu \& Beiser, 2000; Hynie, Guruge \& Shakya, 2012; Qin, Way \& Mukherjee, 2008), criminal and delinquent behavior (Ontario Council of Agencies Serving Immigrants, 2009; Rossiter \& Rossiter, 2009), gang involvement (Rossiter \& Rossiter, 2009) and racism and discrimination (Arthur, Chaves, Este, Frideres \& Hrycak, 2008; Del Villar Nash, 2011; Graham \& Juvonen, 2002; Grossman \& Liang, 2008; Mesch, Turjeman \& Fishman, 2007; Phan, 2003).

First generation newcomer youth may have negative settlement experiences even before reaching Canada. Thomas (2012) explains that the process of leaving their home countries to 
travel to Canada, can result in "profound experiences of disconnections in relationships...Thus, newcomer youth may face isolation as they are without the social supports they had in their homeland and are left without the ties, such as family and friendships that assist with social inclusion" (Thomas, 2012, p. 21-22). In addition, pre-migratory states of war or violence may result in traumatic experiences for newcomer youth and negative psycho-social side effects which may impede their development (Karlovic, 2004; Ngo, 2009, 84).

Once newcomer youth arrive in Canada, they encounter several barriers to successfully settling, integrating and becoming socially included within Canadian society (Karlovic, 2004). Language is often a primary obstacle for first generation newcomer youth as many arrive without knowledge of one or both of Canada's official languages. As well, youth "who arrive in Canada without a solid grasp of their first language may risk losing some aspects of their cultural heritage without access to heritage language support" (Ngo, 2009, p. 84). The Canadian education system is often another barrier for newcomer youth who may struggle adjusting to Canadian curriculum and education systems and making new friends within the classroom (Karlovic, 2004; Ontario Council of Agencies Serving Immigrants, 2009; Quirke, 2011). First generation newcomer youth with low English language skills are disadvantaged when placed in English speaking classrooms in the Canadian education system (Karlovic, 2004; Ngo, 2009; Ontario Council of Agencies Serving Immigrants, 2009; Quirke, 2011). Schools sometimes lack culturally sensitive curriculum, visible minority teaching staff and an awareness of barriers to the participation of newcomer parents within the school system, all of which create obstacles to advocating for change with regards to the education of newcomer youth (Ngo, 2009).

During integration stages, newcomer youth may face barriers in adapting to Canadian or western cultural systems and norms (Ngo, 2009). Feelings of isolation and loneliness may occur 
as the direct result of language barriers, separation from friends and family from their home country and trouble establishing new friendship networks within school and community settings (Hynie et al., 2012; Karlovic, 2004; Quirke, 2011). Newcomer youth may have difficulty acculturating due to experiences of ethnic discrimination (Del Villar Nash, 2011) or being labelled as 'different' based on physical features, name pronunciation, stories of origin or cultural or settlement needs (Karlovic, 2004). Prolonged isolation and loneliness can and prevent newcomer youth from creating co-ethnic friendships or positive mentorships (Ngo, 2009).

Born in Canada, second generation immigrant youth may have difficulty navigating belonging to both Canadian society and their ethnic heritage. Zhou (1997) reports that second generation youth may not have emotional connections to their parents' home country or consider it as a place to visit or reside, therefore, feeling more connected to Canada (Zhou, 1997). Meanwhile, some second generation youth develop a hybrid identity comprised of both Canadian and ethnic values (Gallant, 2008). Pratt (2003/2004) contradicts Zhou's findings writing that issues of belonging for second generation youth are more similar to first generation newcomer youth as "moments of departure and struggle still reverberate throughout their lives, and they continue to feel displaced - not quite at home - in their country of birth" (Pratt, 2003/2004, p. 42). Both authors emphasise that second generation youth may find themselves in a constant struggle, between assimilating into Canadian culture and remaining connected to their parents' cultures of origin (Pratt, 2003/2004) despite the assumption that, because they are born within Canada, they will automatically assimilate with Canadian culture and identity (Pratt, 2003/2004). Khanlou (2008) writes that this struggle with multiple feelings of belonging experienced by second generation youth is due to the fluidity of their identities which have been constructed 
through their experiences within a variety of social contexts, as well as their level of integration within the larger socio-political atmosphere within Canadian society (Khanlou, 2008).

As a function of their age and stage of life, first and second generation immigrant youth grapple with issues of adolescent development such as identity formation, peer pressure and navigating future educational and career pathways (Quirke, 2011). However, first and second generation youth as a whole have more difficulties in education and employment than multigeneration Canadian-born youth and face multiple barriers in accessing support to succeed in these areas (Gonzalez, 2009; Hynie et al., 2012; Phan, 2004; Mesch et al., 2007). Newcomer families, especially racialized newcomer families often have difficulty integrating into the Canadian economy and workforce, resulting in experiences of poverty and segregation defined by impoverished housing and lifestyles due to low incomes (Yan et al., 2008) affecting all members of the family (Omidvar \& Richmond, 2003). The result is an entire population of newcomer youth aged 15-24 having one of the highest unemployment rates for youth in Canada (Ngo, 2009). Despite being born in Canada, second generation youth also face barriers to joining the labour market due to low levels of social capital and fewer networking opportunities outside of their own ethnic community (Yan et al., 2008).

Unemployment and poverty, combined with a lack of access to support services or positive mentors, pre and post migration trauma, social isolation, low language skills, little educational support, and discrimination can result in immigrant youth becoming involved with at-risk or criminal activities for coping, relational or economic reasons (Ngo, 2009; Rossiter \& Rossiter, 2009). Personal and educational support systems are extremely important for both first and second generation youth to prevent them from falling into high-risk lifestyles and to help them with integration. Second generation youth have indicated that friends and family members 
play pivotal roles in supporting their education through providing motivation as well as advice

(Hebert \& Alama, 2008). Ngo (2009) writes that newcomer youth who:

....are in conflict with the law ...experience a wide range of issues in their contact with police and youth justice court procedures. These include distrust and fear of authority figures, limited knowledge about the Canadian justice system, lack of understanding of their constitutional rights, problems understanding and providing accurate information during investigation and court proceedings due to limited English, and cultural misinterpretation (Ngo, 2009, p. 95).

Many racialized first and second generation youth must deal with racism, racial profiling and internalized racism (Ngo, 2009). These encounters with racism or discrimination have been linked to mental health issues (Ngo, 2009). The space in which discrimination occurs varies, however, researchers such as Phan (2004) and Graham and Juvonan (2002) focus on ethnic discrimination occurring within the school system (Phan, 2004; Graham \& Juvonen, 2002) and within Toronto schools newcomer youth "are more likely to report discrimination than nonimmigrant youth" (Del Villar Nash, 2011, p. 18). The perpetrators of discrimination or racism towards newcomer youth include people in positions of authority such as teachers and police and peers of different or similar ethnic backgrounds (Del Villar Nash, 2011; Grossman \& Liang, 2008; Karlovic, 2004; Qin et al., 2008; Ontario Council of Agencies Serving Immigrants, 2009).

Within the literature, often the terms discrimination and bullying are used simultaneously (Qin et al., 2008). Del Villar Nash (2011) however finds that newcomer youth define these terms separately with bullying described as an attack on an individual and discrimination as attack towards an individual or a group (Del Villar Nash, 2011). Valentine and Sporton (2009) also find that British-Somali youth failed to acknowledge racial discrimination when it occurred in public spaces and through laughing off discrimination, they de-racialized their experience and labelled it 'bullying' (Valentine \& Sporton, 2009). Ethnic discrimination can also mobilize 
newcomer youth to seek protection and safety from co-ethnics (Del Villar Nash, 2011) creating situations in which "Some youth have such deep commitment to their ethnic culture that they may socialize entirely within their own ethnic group finding it difficult to integrate into all aspects of life in their new homeland" (Ontario Council of Agencies Serving Immigrants, 2009, p. 19).

Despite being born Canadian, many second generation youth must fight to be recognized as Canadian (Pratt, 2003-2004) and although second generation youth are born, socialized and educated in Canada, they may still struggle with integration, and experience "both short-term and longer-term adjustment issues in reaction to their environment" (Arthur et al., 2008, p. 70). Unlike first generation newcomer youth who may connect to their ethnic culture for protection, for second generation youth, maintaining connections to multiple cultures may develop into "a liability...[as]... Young people realize that to identify with another ethnicity not only risks making them perpetual 'outsiders,' but it may also bring prejudicial and discriminatory action upon them"(Arthur et al., 2008, p. 70). Experiences of discrimination dispute the assumption that the Canadian birthright of second generation youth protects them from such negativity (Arthur et al., 2008) and "some argue that this population may in fact experience higher levels of discrimination than newcomers"(Khanlou, 2008, p. 54-55). Discrimination accompanied by other societal barriers in the areas of employment and education prevent integration of second generation youth while opening pathways to marginalization and isolation (Arthur et al., 2008).

\section{Positive Experiences of First and Second Generation Youth within Settlement, Integration and Social Inclusion}

As discussed above, newcomer youth face a range of negative experiences made up of: 
social, cultural and academic adjustments that are often exacerbated by racism, conflicting cultural values, educational gaps, language difficulties, culture shock, physical health problems, poverty, isolation and/or symptoms of Post-Traumatic Stress Disorder (PTSD) due to war, violence or loss of family members (Rossiter \& Rossiter, 2009, p. 410).

In contrast, some research has focused on the positive experiences of first and second generation youth. Such studies draw on the achievements or positive settlement experiences of newcomer youth within school or home environments and focus on experiences of adaptation, employment, educational achievement, and friendships (Graham \& Juvonen, 2002; Nash, 2011; Phan, 2003; Rossiter \& Rossiter, 2009).

Since the context of each newcomer youth's settlement, integration and inclusion in Canadian society is unique, many experiences may be interpreted as either negative or positive depending on the structural and social environments in which the experience occurred. For example, despite the barriers immigrant youth face within the education system, Rossiter and Rossiter (2009, p. 412) find that "One of the most critical factors in a successful transition to Canadian society is education" and many newcomer youth have positive experiences gaining an education. Educational achievement for both first generation and second generation youth has been linked to factors of assimilation, improved host-language skills, cultural adaptation and family values (Siahaan et al., 2014). A recent longitudinal analysis completed by Siahaan et al. (2014) finds that first and second generation youth have higher educational attainment than native born youth, are more likely to achieve schooling higher than high school and less likely to drop out of high school (Siahaan et al., 2014).

Ethnic identity is defined as "a commitment and sense of belonging to the group, positive evaluation of the group, interest in and knowledge about the group, and involvement in social activities of the group" (Phinney, Cantu \& Kurtz, 1997, p. 168), and the process through which 
newcomer youth develop their ethnic identity is often thought of as a positive experience. Tinkler (2006) found that strong ties to one's ethnic identity can be linked to strong academic performance, whereas Karlovic (2004, p. 42), found that while newcomer youth find value in making Canadian friends, they also feel "that having friends from home their country had helped both themselves and their parents to remain close to their cultural roots and thus benefit from a sense of belonging and inclusion within an extended cultural community of families". Finally, it has also been determined that newcomer youth who are provided opportunities to speak their native languages develop positive cultural identity and language becomes an important cultural characteristic that may be shared with future generations (Karlovic, 2004).

For second generation youth, their ability to navigate between their parents' past migration memories and their current experiences as Canadians gives them a unique perspective on belonging (Pratt, 2003-2004). Kobayashi (2008) summarizes that many second generation youth often:

see themselves and are seen by others as a cultural bridge between their parents' ways of living and a new way of living that is thought of as Canadian. They are agents of sociocultural change, therefore, and a prime locus for understanding the complexities of multicultural society (Kobayashi, 2008, p. 3).

Second generation youth often have multiple layers of belonging which are developed as they locate themselves within different local and global spaces (Hebert, Wilkinson \& Ali, 2008). Due to their multiple layers of belonging "The second generation is the most likely of all generation groups to value Canada for its multiculturalism and diversity" (Hebert et al., 2008, p. 64). This duality does not limit or trouble most second generation youth but instead they are comfortable with multiple senses of belonging and identity, accept individual differences(Hebert et al., 2008) 
and become engaged "in transcultural processes of creating new modes of belonging" (Hebert et al., 2008, p. 66-67).

Research by Ali (2008) describes second generation youth having positive experiences with multiculturalism. From the youths' perspectives, multiculturalism is a tool that empowers their families to leave situations of poverty, inequality and violence. Multiculturalism facilitates spaces to interact with people from a variety of cultural identities and upholds a "feeling that they were not judged by their peers on the basis of their race, religion or ethnicity and that diverse people got along fairly well in Canada" (Ali, 2008, p. 87-88). However, in this specific study, the youths' positive identification with multiculturalism was limited to "their multicultural schools and neighbourhoods...[where] they could claim their multiple identifications with a sense of confidence" (Ali, 2008, p. 89). Outside their neighbourhoods, the youths' experiences of multiculturalism were overshadowed by the visible barriers and negative labels placed on the shoulders of immigrants and their communities (Ali, 2008). Second generation youth's ability to negotiate multiple senses of belonging and identity has allowed them a unique position to analyze and critique their positive and negative experiences, and as "These young people reflect on multiculturalism and democracy, finding them both laudable for their human rights, but mostly also decrying the shortcomings of multiculturalism and democracy, as there is still racism and discrimination in Canada" (Hebert et al., 2008, p. 68).

\section{Similarities and Differences between Multicultural, Mainstream and Ethno-specific Community Organizations}

First and second generation immigrant youth access services from a variety of multicultural, mainstream and ethno-specific organizations during their settlement, integration 
and inclusion phases. Research on service provision for newcomers usually focuses on organizations labelled as settlement organizations. Some organizations are labelled settlement organizations because they provide services exclusively to newcomers. For other organizations, it is their funding source that determines that title (Lim et al., 2005) as they receive federal or provincial funding directed towards addressing the settlement and integration needs of newcomer youth and their families (Lim et al., 2005; Thomas, 2012).

Many organizations may not receive settlement specific funding nor provide settlement focused services but still serve newcomer clients; therefore, there is a need to expand the understanding of which organizations fall under the settlement definition (Lim et al., 2005). In 2005, Lim conducted a survey of organizations working with newcomers and found 101 ethnospecific organizations and 226 settlement organizations within the city of Toronto, however his study did not take into consider the services provided to newcomers from mainstream organizations (Lim et al., 2005). Mainstream, multicultural and ethno-specific organizations provide services to newcomer clients in unique ways, with each type of organization exhibiting different characteristics.

\section{Characteristics of Multicultural Organizations}

Multicultural organizations intentionally focus on providing services to diverse immigrant populations and they are usually the organizations receiving federal 'settlement' service funding designed to support services for newcomers within their first three years. Attending community youth programs within "Multicultural settings provide opportunities for becoming aware of one's cultural identity, not only in contrast to a dominant majority, but through ongoing contact with other cultures" (Khanlou, 2008, p. 55). Multicultural organizations 
also provide environments in which first generation newcomer youth are free to identify with their ethnicity or culture in a multitude of ways (Ali, 2008). Within a multicultural setting, youth may choose a range of racial, religious, ethnic or cultural identifications or they may choose "to establish their unique group memberships" (Ali, 2008, p. 87). Many multicultural organizations are not funded to provide services to newcomer youth after their first three years, with the general belief that newcomer youth are settled enough in Canada to access mainstream services.

\section{Characteristics of Mainstream Organizations}

Despite many newcomer youth attending mainstream services, mainstream organizations sometimes are not prepared to address their unique social and cultural needs (Ngo, 2009). Ngo (2009) argues for mainstream organizations to increase their cultural competence as "[c]ultural competence requires organizations to explicitly recognize and integrate cultural diversity into all aspects of organizational structures and functions" (Ngo, 2009) which will ameliorate newcomer youth access. Ngo discovered that mainstream organizations such as the YMCA and the Boys and Girls Clubs integrate varying levels of cultural competency including policies focusing on inclusion and diversity and programs designed specifically to meet the needs of newcomer youth; however, newcomer youth participation remains proportionately low (Ngo, 2009). To remedy this, cultural competence cannot exist as an additional program or policy but instead must be fully integrated within mainstream organizations' governance, policies and programming (Ngo, 2009).

Mainstream organizations serve youth from a variety of backgrounds including 'Canadian', 'Aboriginal' or 'Newcomer' in identity. This integrated recreational environment provides opportunities for newcomer youth to be adventurous while sharing their culture with 
peers from the dominant ethnic group (Tirone \& Pedlar, 2005). However, mainstream organizations can be places of acute stress due to discrimination from dominant ethnic groups (Tirone \& Pedlar, 2005). To avoid negative mainstream recreational experiences, newcomer youth may "seek out leisure experiences with people from their homeland, thus perpetuating and solidifying the experience of...leisure within ethnic enclaves" (Tirone \& Pedlar, 2005, p. 35) or ethno-specific organizations. However, for other newcomer youth, participating in multicultural recreational settings provided them with an opportunity to celebrate their diversity as well as bond over shared experiences of mainstream discrimination (Gonzalez, 2009; Tirone \& Pedlar, 2005). This form of bonding is referred to by Wu, Schimmele and Hou (2012) as 'situational solidarity' (Wu, Schimmele \& Hou, 2012).

\section{Characteristics of Ethno-specific Organizations}

Research on ethno-specific organizations is often dedicated to understanding why individuals choose to participate in or develop ethno-specific services. The creation of such homogeneous ethnic communities is often explained as "a defensive maneuver against discrimination and racism" (Wu et al., 2012, p. 21) within the dominant culture/society. Wu, Schimmele and Hou (2012), find that within ethnically homogeneous communities, individuals are more trustful of each other due to stronger interconnections built between them $(\mathrm{Wu}$, Schimmele \& Hou, 2012). Vo-Jutabha, Dinh, McHale, and Valsiner (2009) add that within ethnic enclaves, youth have a stronger sense of their ethnic identity compared to youth who live outside of ethnic enclaves and may sway their cultural identities for mainstream culture (VoJutabha, Dinh, McHale \& Valsiner, 2009). Ethno-specific organizations can often be thought of as microcosms of ethnic enclaves and thus exhibit some of the same characteristics. Within ethno-specific organizations, workers often share similar ethnic and immigration backgrounds to 
the youth participants and such commonalities "may influence their perceived helpfulness or trustworthiness among newcomers" (Quirke, 2011, p. 350).

\section{Relationship between Community Programming and Immigrant Youth}

Immigrant youth interact and exist within home, school and community environments (Ngo, 2009). Community environments offer services to first and second generation youth through non-profit organizations, religious institutions and educational institutions (Thomas, 2012). Zhou (1997) writes that "levels of adaptation among young immigrants are generally measured by educational attainment, academic orientation, aspiration, and performance" (Zhou 1997, p. 75), therefore the community environment in which newcomer youth spend their time outside of school, is left unrecognized for its impact on adaptation, integration and social inclusion. Whereas many studies research the experiences of newcomer youth within school or home environments, few are conducted within the community environments focusing on services such as community youth programming (Graham \& Juvonen, 2002; Nash, 2011; Phan, 2003). Research on community youth programming demonstrates many benefits for youth participants from the general population, however, it is lacking in research focused on the relationship between community youth programming and immigrant youth (Greenberg, 2013). Community youth programming provides a diverse range of activities for newcomer and Canadian youth dependent on specific program/project goals, funding requirements, space and environmental factors as well as demand and interest.

There are many reasons why young people attend community youth programming. Community organizations help youth with their homework and allow them to experience a variety of creative and athletic programming in which youth become connected to larger social 
supports within their communities (Greenberg, 2013; Rossiter \& Rossiter, 2009). The additional academic support youth receive at community youth programming is very important (Fokkena, 2011; Greenberg, 2013). Youth participants are provided opportunities to construct skills physically, socially, emotionally and cognitively (Greenberg, 2013). Long-term effects of afterschool programming are "associated with higher than expected grades, higher self-esteem, resiliency, and lower than expected risky behavior" (Greenberg, 2013, p. 102).

For other youth, community youth programming is a space in which they receive positive behavior incentives (Greenberg, 2013). Children and youth involved in after-school programming are surrounded by "positive behaviour-reinforcing peers" (Greenberg, 2013, p. 102) aiding their "development and growth" (Greenberg, 2013, p. 102; Herzog, 2011, p. 229). Community programs also promote protective factors such as "pro-social inter-cultural peer programmes and relationships" (Rossiter \& Rossiter, 2009, p. 421) which may prevent newcomer youth from criminal involvement. Many criminally involved youth are excluded from accessing positive programming within schools and therefore community programing may be their last opportunity to develop positive connections within a mentoring environment (Ngo, 2009). Without access to community programming that provides youth with opportunities to connect, youth may become isolated and may partake in criminal activities (Herzog, 2011).

Youth may also attend community youth programs for support with acclimatization and adaptation (Ngo, 2009; Thomas, 2012). Karlovic (2004) in her interviews with newcomer youth found that "Many of the positive adjustment experiences identified by the youth informants centered upon involvements with sports, music, and other extracurricular school activities" (Karlovic, 2004, p. 68). Tinkler's (2006) dissertation on the expression of freedom of refugee youth examined a photo-based youth program activity, revealing that through this program, 
refugee youth within the USA were able to "challenge and appropriate American discourses of freedom" (Tinkler, 2006, p. 166) thus negotiating their place within American society.

Recreational activities, such as sports, are one type of activity offered within community youth programming and play an important role in the settlement and integration of first and second generation newcomer youth. Karlovic (2004) found that newcomer youth describe sports as important aspects of their culture and that male newcomer youth often show "pride in their athletic activities in Canada which they associate with their cultural backgrounds and experiences in [their home country]" (Karlovic, 2004, p. 51). Through participating in sports, newcomer youth create friendships, increase self-esteem and gain confidence in social and community interactions thus facilitating their integration and social inclusion (Karlovic, 2004). Tinkler (2006) adds that though community programs cannot change traumatic experiences of newcomer families, they can offer a space where newcomer youth can develop resilience.

Lastly, many youth find that participating in community youth programming helps them foster connections with their community (Tinkler, 2006; Thomas, 2012). Within Community Youth Programs, youth develop a network of friends and positive adult support (Greenberg, 2013) and Zhou (1997) explains that newcomer children who are involved in "tightly knit" social networks may have better psychological wellbeing, academic achievement and understanding of future education or careers than those newcomer youth who experience social isolation (Zhou 1997). Community organizations provide a special space for newcomer youth often acting as "as safe passages between schools and communities" (Tinkler, 2006, p. 4). Fine, Weis, Centrie and Roberts (2000) define community youth programming as a 'space of difference' where marginalized individuals within a community location are provided space to "sculpt real and imaginary corners for peace, solace, communion, personal and collective identity work" (Fine, 
Weis, Centrie and Roberts, 2000, p. 132). Newcomer youth are often marginalized within their communities, however, youth programs offer them a safe environment to develop a sense of belonging and identity (Hebert et al., 2008; Tinkler, 2006) while contesting "the stereotypes and negative messages they may receive in their new communities" (Tinkler, 2006, p. 4, 40). Newcomer families are more likely to send their children to community programs because they "provide a sense of extended family and safe supervision for the children in their communities" (Rossiter \& Rossiter, 2009, p. 423). Rossiter and Rossiter (2009) also state that programs allow newcomer youth to give back to their communities by fostering leadership skills and self-esteem.

Community organizations create a space of social inclusion. Thomas (2012) writes that "community outreach by settlement organizations diminishes social exclusion from wider society and sense of belonging to the larger community is enhanced" (Thomas, 2012, p. 20). This is a product of community programming focused on providing newcomers a chance for networking and social interaction (Thomas, 2012). Community youth programming is a great source of valuable information which connects newcomer youth and their families "to housing, health care, education, and employment, and facilitate [their] subsequent inclusion into the social and political fabric of Canadian life" (Quirke, 2011, p. 347-348). Staff and volunteers working in these programs become mentors for newcomer youth, providing them with knowledge on education, health, finances, positive communication, conflict resolution and day to day advice (Rossiter \& Rossiter, 2009). Tinkler (2006, p. 39) writes that "the most successful programs maintain strong ties with the community and are culturally consistent with the population they serve". In addition, because, "As many as $51 \%$ of immigrants under the age of 15 and $41 \%$ of those aged 15-24 live in poverty" (Ngo, 2009, p. 85) many community youth programs provide their services to newcomer youth for little to no cost. 
Despite the many benefits associate with community youth programs, there also some common struggles experienced by community organizations in providing youth programming. One struggle is that some community organizations provide programming that is not culturally inclusive, thereby creating cultural barriers to the participation of newcomer youth (Ngo, 2009). Cultural barriers combined with the limited knowledge of newcomer families regarding community level services contributes to overall low numbers of newcomer families participating in community programs $(\mathrm{Ngo}, 2009)$. In Canada the overall number of newcomer youth who participate in community or after-school programming is unknown, however in America, one study found that less than ten percent of American newcomer youth stated that they were involved in after-school programs (albeit, academic focused) (Greenberg, 2013). Karlovic (2004) found that, in Canada, almost 1/3 of newcomer youth interviewed had never heard of community youth programming, whereas $50 \%$ of those interviewed stated they did not have time or did not access such services. Based on American studies, Greenberg (2013) connects such low attendance to more youth spending after-school time with family, low parent education, income and the age of the child. Greenberg (2013) also found that racialized children are less likely than Caucasian-European children to participate in afterschool-activities which may be a result of discrimination occurring within youth program and school environments (as cited in Mesch et al., 2007).

In summary, newcomer youth search for and attend programming that "[facilitate] positive connections to place, promoting well-being and contributing to new arrivals' becoming at home in their country of resettlement" (Sampson \& Gifford, 2010, p. 116-117). In the areas of research on newcomer youth and community programming three Canadian studies have contributed to promising research. First, Ontario Council of Agencies Serving Immigrants 
(OCASI) in collaboration with newcomer youth published their best practices for community programs working with newcomer youth. The list states that community organizations should provide "a wide range of activities...ensuring...[they] serve newcomer youth from diverse communities...[and help] youth and parents deal with exclusion and racism in school and in other aspects of life" (OCASI, 2009, p. 7). It is recommended that Settlement services adopt antioppression or anti-racism frameworks to ensure that such principles and practices are incorporated into programs (OCASI, 2009). OCASI also requests that youth should face no exclusion from services due to their skin colour or ethnicity, that staff should receive diversity training, and that "Programs [should] not tolerate any form of racism or discrimination, [ensuring] a safe and respectful space for youth" (OCASI, 2009, p. 34).

A second study by Ngo (2009), analyzes youth specific settlement services comprised of both multicultural and mainstream organizations in Calgary, Vancouver and Toronto and their ability to address the settlement and integration needs of newcomer youth. Ngo (2009) ultimately finds that the organizations he studied help newcomer youth adapt to Canadian society, but fail to address deeper concerns such as cultural identity and trauma. As these organizations do not intentionally provide opportunities for newcomer youth to integrate into the larger community they are "risking reinforcing over-reliance of immigrant youth on intra-ethnic or immigrant networks" over community networks (Ngo 2009, p. 89). Ngo (2009) reports that newcomer youth go through an evolution of change from arrival to 'integration', and suggests that services should be located throughout the continuum to serve different needs of youth as they go through the process. Presently, Ngo (2009) notes that youth programs focusing on advocacy or leadership within the integration phase are limited, and do not help newcomer youth 
socially locate or critically question their political, social or cultural reality or the racism and discrimination that many youth face daily.

Ngo (2009) suggests that organizations actively involve newcomer youth in program development and evaluation and for increased community advocacy to promote funding and "equality and equity in service delivery to children of immigrant families" (Ngo, 2009, p. 95) because "immigrant youth are entitled to services that effectively address their settlement needs and promote their full participation in Canadian society" (Ngo, 2009, p. 85). Ngo's analysis is thorough in its overview of programs accessed by newcomer youth, but it is lacking in its depiction of the personal experiences of participating staff and youth. It is difficult to analyze community youth programming in relation to its intended impact on settlement of newcomer youth because, as Ngo found, many programs do not publicly link the issues they work on to the acculturation of newcomer youth nor the theoretical perspectives behind their form of service delivery (Ngo, 2009). Thus Ngo $(2009$, p. 88$)$ concludes that "the existing service delivery to immigrant adolescents appears intuitive and less coherent than planned, evidence-based practice".

A third study by Thomas (2012) was conducted within Greater Toronto Area (GTA) examining settlement services (multicultural organizations) and their ability to remove barriers of social exclusion for newcomer youth. Thomas argues that in order to successfully integrate newcomers into Canadian society, newcomer youth must be able to connect with socially inclusive environments (Thomas, 2012). Thomas' study looks at the experiences of newcomer youth within youth settlement services. She finds that youth attend settlement program to learn English, to gain volunteer hours, to receive homework help or hang out with friends. Youth participants in Thomas' study describe settlement services as creating a family-like environment 
with both recreational and educational opportunities. Youth also describe having strong relationships with program staff (Thomas, 2012). Although Thomas' study increased knowledge regarding positive experiences of newcomer youth with services, newcomer youth "unanimously explained that they had no negative comments to make about existing programs" (Thomas, 2012, p. 45) which may have been more a result of the focus group environment than the quality of programming. However, Thomas' study is significant because it connects settlement youth programming to fostering social inclusion in the lives of newcomer youth in five areas: Relational Inclusion, Labour Market Inclusion, Spatial Inclusion, Educational inclusion, and lastly Socio-political inclusion (Thomas, 2012). 


\section{THEORETICAL FRAMEWORK}

Laidlaw Foundation's (2003) social inclusion framework will be employed in the analysis of the findings of this study. Currently, social inclusion is seen as an ideal end goal for communities comprised of newcomers and mainstream Canadians. Social inclusion is therefore an appropriate theoretical framework to analyse the positive and negative experiences of first and second generation youth within mainstream, multicultural and ethno-specific community youth programming and evaluate the extent to which such programs provide experiences of social inclusion or exclusion of newcomer youth. Thomas (2012) presented evidence that multicultural organizations provide the space and tools for first generation newcomer youth to feel included and build towards inclusion in the larger society. Other research shows that first and second generation youth still struggle to be included in Canadian society. The theoretical section of this study will examine social inclusion as a concept, then review research which connects social inclusion to newcomer experiences and finally describe Laidlaw Foundation's (2003) social inclusion framework in detail.

\section{Social Inclusion as a Concept}

Social inclusion was introduced in the 1980s and 1990s as a "vehicle to enhance access and equity in the field of social policy and programming" (Omidvar \& Richmond, 2003, p. 10); however, it has expanded to cover areas such as bridging societal divides, promoting participation and creating shared experience. Equity and access are essential to the definition of social inclusion, as Duncan (2003) writes that socially inclusive societies provide equal access for everyone to achieve the 'good life' (Duncan, 2003). Saloojee (2003) further advances the relationship between social inclusion and equality by explaining that social inclusion extends 
beyond 'formal equality' into 'substantive equality' which involves confronting forms of exclusion and inequality (Saloojee, 2003, p. 9).

Active community participation of newcomers is vital to the definition of Social Inclusion. Omidvar and Richmond (2003, p. 1) write that "For immigrants and refugees, social inclusion would be represented by the realization of full and equal participation in the economic, social, cultural and political dimensions of life in their new country". Caidi and Allard (2005, p. 313) support the importance of participation because "It seems essential...that people have a voice in what they are included into. Involvement and engagement expresses the right and support of individuals to make meaningful decisions about themselves and their community".

Social inclusion is a means to building 'common ground' which will lead to the participation in society of ethno racial communities (Omidvar \& Richmond, 2003, p. 10). Duncan (2003) suggests that building common ground between groups of immigrants and Canadian born, aids in breaking down the racialized barriers of poverty and links social inclusion to discussions of racism and anti-racism (Duncan, 2003). Saloojee (2003) adds that social inclusion promotes "...a transformative agenda that links together the various, often disparate struggles against oppression, inequality and injustice" (Saloojee, 2003, p. 1). Barriers are removed and reduced by confronting the physical, social or economic barriers which separate those labelled as privileged and those labelled as marginalized (Omidvar and Richmond, 2003).

The strengths of Social Inclusion include its flexibility and its focus on community involvement and participation. Social inclusion purposefully targets the participation of minority and ethnic groups through both its practice and policy creation. Guo and Guo (2012) emphasise that in order: 
To build an inclusive society, it is imperative to treat ethnic community organizations as an integral part of Canadian society and to adopt minority rights that recognize and accommodate the distinctive identities and needs of ethnocultural groups and their ethnic communities (Guo \& Guo, 2012, 5).

Social inclusion acknowledges the importance of difference and diversity (Omidvar \& Richmond, 2003). It "calls for a validation and recognition of diversity as well as a recognition of the commonality of lived experiences and the shared aspirations among people, particularly evident among families with children" (Omidvar \& Richmond, 2003, p. ix). In promoting participation, Saloojee (2003) argues that social inclusion builds much needed solidarity among people of diverse backgrounds, between host communities and immigrants to work together in creating inclusive communities (Saloojee, 2003).

There are also convincing arguments regarding the weaknesses of social inclusion. First, Omidvar and Richmond (2003) note that the definition of social inclusion is extremely flexible (Omidvar \& Richmond, 2003). As stated above, this can be positive, but it can also be a weakness when formed by political beliefs of the day resulting in a Ponzi term that never influences practice (Omidvar \& Richmond, 2003). Caidi and Allard (2005) raise concern about social inclusion leading to government policies of assimilation. To prevent this, they insist that Canadians facilitate the integration of immigrants (Caidi \& Allard, 2005). Saloojee (2003) agrees with Caidi and Allard that the weakness of social inclusion is its vagueness and its frequent association with assimilation. He questions whether social inclusion is multiculturalism in disguise, a new way of managing minority relations at a government level (Saloojee, 2003). Duncan (2003) argues that one could see social inclusion as “...making everyone worse off for the sake of greater equality for all" (Duncan, 2003, p. 2) because inclusion results in the 'good life', a scarce commodity being over extended to immigrants resulting in less 'good life' for those who had it before (Duncan, 2003). 


\section{Social Inclusion and the Immigrant Experience}

The terms settlement, integration and social inclusion are often used in research to describe the trajectory from physical arrival in Canada to emotional, societal and economic inclusion into Canadian society. Settlement, defined as "the process by which newcomers to a country, including both immigrants and refugees, orient themselves to their new homelands" (Quirke, 2011, p.346) includes the fulfillment of immediate needs such as shelter, employment, and food. The process of settlement "may refer to the experiences of the first few weeks, months, or years in a new country, depending on the context in which it is used" (Quirke, 2011, p. 346). Integration follows settlement when newcomers become involved in services which develop or build on various skills needed for integration into employment, education and Canadian culture and society. After integration, newcomers may reach social inclusion which is "the long- term struggle for equal participation in all realms of life, politically, socially, economically and culturally" (Quirke, 2011, p. 346). Reaching social inclusion can take years, and sometimes generations as some question whether second generation youth have attained social inclusion. Quirke (2011, p. 347) adds that social inclusion "involves the eventual dismantling of all systemic barriers to participation of an immigrant group and their descendants within society, and is a process that can take multiple generations or even centuries to achieve".

Community organizations or settlement services have the potential to aid newcomers in social inclusion, however they often are constrained by their funding and thus the impact of their services may be limited. Omidvar and Richmond (2003) write that:

One of the most serious problems of the current system lies in the fact that settlement funding and programming is focused on the initial stages of adaptation, in spite of the fact that the process of settlement continues throughout the life of the newcomer (Omidvar \& Richmond, 2003, p. 8). 
In fact, assistance with social inclusion often occurs "[i]n the long term or final stage of settlement, [where] immigrants and refugees strive to become equal participants in Canada's economic, cultural, social and political life" (Omidvar \& Richmond, 2003, p. 8). Funding is directed toward short-term projects rather than longer-term core funding placing stress on smaller organizations to address the diverse needs clients, many of whom may no longer fall under their federally identified service population (Omidvar \& Richmond, 2003). It is necessary to redefine the concept of settlement acknowledging that "[t]he settlement journey for newcomers is one that lasts a lifetime and extends into the second generation" (Omidvar \& Richmond, 2003, p. 18) as well as recognizing that the social inclusion of newcomers is a joint responsibility and mutually beneficial for newcomers and the receiving Canadian society. To reach such socially inclusive policies, community organizations who serve immigrants must be recognized and protected for their important services and role in advocacy and the voices of newcomer youth themselves must define and oversee all policies, programming, research and evaluation processes implemented (Omidvar \& Richmond, 2003).

Thomas' (2012) study connects settlement youth programming to fostering social inclusion in the lives of newcomer youth in five areas: Relational Inclusion, Labour Market Inclusion, Spatial Inclusion, Educational Inclusion, and lastly Socio-political Inclusion. Relational Inclusion is fostered through the development of new friendships, the maintenance of existing friendships, the opportunity to connect with youth of different cultural backgrounds and the development of trusting relationships with the families of participating youth. Thomas writes that "participants emphasized that if they had not attended the program, they would have been less likely to form connections made outside their own cultural and ethnic background, as there is less motivation to form these connections" (Thomas, 2012, p. 50). 
Labour Market Inclusion is fostered in settlement services as these services build employment and leadership skills of newcomer youth through workshops and training opportunities. Spatial Inclusion is facilitated by settlement services in fostering connections between newcomer youth and local neighbourhoods. Educational Inclusion is fostered through helping with homework and providing post-secondary education guidance. Youth settlement services are found to create opportunities of socio-political inclusion for newcomer youth as they provide volunteering experiences and involve many newcomer youth in program development internally preparing youth for a future of critical involvement in their communities (Thomas, 2012).

\section{Social Inclusion as a Theoretical Framework}

In order to analyse the experiences of first and second generation immigrant youth within community youth programming through a social inclusion lens, it is necessary to provide a concrete framework of social inclusion. The Laidlaw Foundation's (2003) social inclusion framework, underlying their working paper series on social inclusion will be used to further unpack the impact of community youth programs in creating an environment of social inclusion for first and second generation newcomer youth. Laidlaw's (2003) framework of Social Inclusion consists of five cornerstones.

The first cornerstone, Valued Recognition, insists that social inclusion involves giving respect to individuals and groups. Within multicultural, mainstream and ethno-specific organizations are youth providing examples of being respected and giving respect to others? The second, Human Development, refers to "Nurturing the talents, skills, capacities and choices of children and adults to live a life they value and to make a contribution both they and others find 
worthwhile" (Omidvar \& Richmond, 2003, p. vii ). Within the multicultural, mainstream and ethno-specific organizations, are youth provided with positive experiences which develop their skills, passions and leadership skills? The third cornerstone, Involvement and Engagement, provides people the opportunity and support to participate in community decisions that affect their life. Do multicultural, mainstream and ethno-specific organizations provide opportunity for community connections within their programming? The fourth, Proximity, is providing shared space to create positive interactions between people and decrease negative social distance between groups. Does community youth programming connect people of diverse backgrounds and work actively to address barriers of exclusion both within the programs and within the community? The last cornerstone, Material Well-Being, is describes as providing resources or tools for individuals to participate and succeed within the community (Omidvar \& Richmond, 2003). Do mainstream, multicultural and ethno-specific organizations provide information, mentorships and other tools necessary to help youth overcome barriers to social inclusion? These five cornerstones will facilitate an analysis of the level of inclusiveness community youth programs experienced by first and second generation newcomer youth. 


\section{METHODOLOGY}

\section{Research Approach}

Due to the limited base of knowledge regarding the experiences of first and second generation newcomer youth within community youth programming, research for this study was conducted using a qualitative exploratory research design. Exploratory research is essential when there exists "little or no scientific knowledge about the group, process, activity, or situation they want to examine but nevertheless have reason to believe it contains elements worth discovering, (Stebbins, 2001, p. 6). This study seeks to determine: What are the services provided for newcomer youth through mainstream, multicultural and ethno-specific programming? What are the positive and negative experiences of immigrant youth within community youth programming? And, what are the youths' recommendations to improve community programs? From my own experience, I have seen the rich and vibrant impact such programs have on newcomer youth and through utilising an exploratory approach I hope this study lays the foundation for further research to be conducted on this relationship.

The development of a methodology to explore the experiences of first and second generation youth within community youth programs began with questioning my own assumptions regarding youth research and how I should interact with youth and community organizations within a research relationship. Greig, Taylor and MacKay (2007) state that it is important to question and challenge one's assumptions regarding youth and childhood when conducting research with adolescents. A researcher's perception of adolescence is often derived from personal experiences, professional training, culture and one's identity (Greig et al., 2007; Temple \& Edwards, 2006). My personal perceptions of youth and adolescence are primarily 
derived from my professional experience working with youth within a community organizational environment. I strongly disagree with the assumption that youth are "not able to contribute reliably towards discussions on their feelings, needs and future" (Greig et al., 2007, p. 89). In contrast, I have found youth to be valuable participants in research and "[l]istening to children can give...insight into the child's mind that [researchers] could not otherwise have" (Ceglowski \& Makovsky, 2012, p. 285).

Initial methodological selections regarding data inclusion, methods and interview questions were developed based on best practices for involving youth research participants and feedback provided from the university's Research Ethics Board. Working from a social inclusion lens, I worked under the assumption that it is essential to include the voices of youth and service providers as active research participants, as only they can contribute the information needed to identify the positive and negative experiences within community youth programming. As a researcher, I walk the delicate methodological balance between respecting the ethical considerations required by the university research ethics board, and addressing and valuing the research needs of the participating organizations and youth.

\section{Research Setting}

Toronto, with its ethno-cultural diversity, newcomer concentration and unique settlement distribution patterns, provides a rich environment for the study of first and second generation youth experiences within community youth programming. Toronto has become one of "the most ethnically heterogeneous places in the world" (Wu et al., 2012, p. 5) which has facilitated the growth of ethnic enclaves and the formation of ethno-specific settlement organizations (Meinhard \& Faridi, 2009, p.1) providing a unique youth programming setting. Approximately 
half of Toronto's population is comprised of immigrants and, therefore, "the need for effective, accessible newcomer services in the Toronto city-region is great" (Lim et al., 2005, p. 4). In addition, children of immigrants under age 24 represent approximately $37 \%$ of permanent resident population (Ngo, 2009) and 20\% of all children in Canada under the legal age of 18

(Arthur et al., 2008). Historically, settlement in Toronto existed primarily in the downtown core, but now has spread to the suburbs, primarily North York and Scarborough, and the outer cities of the Toronto Census Metropolitan Area, Markham, Mississauga, Brampton and Vaughn (Lim et al., 2005). In my study I attempt to represent community organizations in a variety of these areas, looking at one situated in downtown, and two in North York.

\section{Research Design}

I have chosen to use a qualitative research design for its ability to "allow the researcher the greatest insights into group dynamics and individual motivations" (Archer \& Berdhal, 2011, p. 133) during exploratory research. Qualitative research is often used to provide answers to research questions by looking at "how humans arrange themselves and their settings and how inhabitants of these settings make sense of their surroundings" (Berg, 2009, p. 8). As Thomas (2012) writes, "This method allows this marginalized group to have their voices heard and livedexperiences shared" (Thomas, 2012, p. 29). The specific qualitative tools utilized within this study are semi-structured and structured interviews and a demographic questionnaire. Copies of the interview guides and demographic questionnaire are located in Appendix A.

Much thought went into selecting the tools used to gather information from both staff and youth research participants. Semi-structured interviews were chosen for the nine youth participants as they are structured to maintain flexibility for the direction of conversation to be 
altered with probes in response to topics brought up by the youth participant (Berg, 2009). The semi-structured interviews utilized within this study contained five main structured questions followed by several unstructured probes that were used to draw out youth's responses. Using a semi-structured interview structure allowed for questions to be developed in line with my research questions, while allowing space for youth participants to be relaxed and express themselves normally (Vega, 2011). Semi-structured interviews also allowed for adaptation of the vocabulary used within the interview to meet the language level of the participants which was advantageous since some of the youth were recent arrivals to Canada (Berg, 2009).

Structured interviews were used for three staff interviews because the purpose of the staff interviews was to primarily uncover factual information regarding the three different organizations and the programs they provided to first and second generation youth. The structured interviews were fourteen questions, with no probes. Structured interviews "offer each subject approximately the same stimulus so that responses to questions, ideally will be comparable" (Berg, 2009, p. 105) and would provide a good data base to compare and contrast the services offered between the mainstream, multicultural and ethno-specific organizations. Lastly, a short demographic questionnaire which comprised of questions regarding the youth and staff's gender, age, ethnic identity and immigration status was used to provide demographic background on the interview participants.

\section{Research Participant Recruitment}

To explore further the experiences of first and second generation newcomer youth within mainstream, multicultural and ethno-specific youth programming it was necessary to recruit youth participants from all of these types of organizations in Toronto. To do this I used 
purposive sampling techniques to compile a list of newcomer youth serving agencies, with the help of the Ontario Government Newcomer Settlement Agencies by Location list (http://www.citizenship.gov.on.ca/english/newcomers/agencies.shtml) and United Way Toronto's list of community agencies (http://www.unitedwaytoronto.com/list-of-agencies\#A). Purposive sampling, allows for the researcher to identify "cases that will provide the greatest amount of information" (Archer \& Berdahl, 2011, p.173) thus narrowing the list of newcomer youth serving agencies to those agencies who displayed mainstream, multicultural and ethnospecific characteristics. To locate one mainstream, one multicultural and one ethno-specific organization to participate as a recruitment base of youth and staff participants, I e-mailed all of the organizations explaining my research questions and intent to interview one staff and three youth participants from their youth programming. Three organizations, representing the three types of organizations of interest to my research, accepted my request and facilitated an opportunity for me conduct a recruitment presentation within their youth programs. Documents used within the recruitment phase of this study are located in Appendix B.

The recruitment presentation was delivered within the youth programs of a mainstream, a multicultural and a ethno-specific community organization. If the host organization had multiple youth programs, they selected which program I would do the presentation for. The recruitment presentation was ten minutes in length and covered topics such as: defining research, information on my research questions, the recruitment, interview and consent processes, research participant rights, participation incentives and lastly instructions on how each youth could volunteer to participate as it is important for the researcher to explain the purpose of the study and go step by step through the research process with the youth (Greig et al., 2007). The language used within the presentation, the consent forms and the interview questions was adapted to match the 
cognitive and English language ability of prospective youth participants so that they would understand the research questions, processes and be able to give their own consent to participating (Due, Riggs \& Augoustinos, 2014). Through the recruitment presentation, I sought to recruit nine first generation newcomer youth (three from each participating organization) between the ages of 12 and 18. During the recruitment stages, many second generation newcomer youth contacted me to participate, therefore resulting in the expansion of my research focus to include the experiences of second generation youth as well. Also, the age range was expanded to include youth up to 24 as some of the youth program involved catered to youth 1424 years old, thus impacting how I defined 'youth' within this study.

Youth were provided with two confidential methods to volunteer to be interviewed. Both methods relied on the youth present for the recruitment presentation to take initiative in volunteering for the interviews. Both methods also were implemented with the objective of protecting the identity of those youth who volunteered which despite making it more difficult for researchers to recruit youth, protected the personal information of youth who did not wish to participate in the study (Greig et al., 2007). The first recruitment method was a handout provided to all youth who attended the recruitment presentations. The recruitment handout included a summary of the research study as well as instructions on how to contact me to volunteer for an interview. This technique was not successful as only two youth contacted me through using this method.

The second recruitment method used was an opt-in form which was also provided to all youth participating in the recruitment presentations. To indicate they were interested in participating in the interview the youth would write their first name, either a phone number or email and check a box stating that they were interested in being contacted regarding an 
interview. Youth who did not want to participate would leave the form blank. All youth would then fold their forms in half, concealing their participation status and one youth would collect and submit the forms to me. From the interview opt-in forms, up to three youth were randomly chosen from each organization and then contacted to follow-up on their interest in being interviewed. If a youth was no longer interested, their opt-in was destroyed and another was randomly selected from the pile. Once all three participants were confirmed with set interview dates, all other opt-in forms were destroyed. This form of recruitment was very successful as I recruited seven out of nine youth participants in this manner.

During the recruitment phase, it was important to create an atmosphere of confidentiality as Grieg et al. (2007) find that youth "may be concerned that their responses can be accessed by those who have power over them and consequently may expect to receive poorer care, support and understanding" (Greig et al., 2007, p. 92). To create an atmosphere of confidentiality, organizational staff members were asked to not be present during recruitment presentations, youth's participation in the interviews was not disclosed with the participating organizations and the youth's responses have been protected by a pseudonym chosen by each participant within this study.

Due and associates (2014) suggest that when conducting research with youth, it is important to spend time with them before the interview in order to foster a connection and create trust between researcher and participant (Due et al., 2014). Although I faced time limitations for conducting interviews, I chose to do recruitment personally through recruitment presentations so that youth would be able to see me and learn about my project in person and have any questions they have answered in person. I believe this small step facilitated a better interview relationship as youth interested in being interview were often contacted back by phone and I could hear the 
element of recognition when I re-introduced myself which allowed them to feel more comfortable arranging an interview.

Recruitment processes for the staff interviews was very different than for the youth interviews. I sought to recruit one staff member directly involved with youth programming from each the mainstream, multicultural and ethno-specific organization. Participating organizations were informed of the need for one of their youth program staff to be interviewed and were asked to provide the researcher's contact information to their staff if they were interested in being interviewed. Interested staff made contact with the researcher either in person before or after the recruitment presentation or through email. The participating staff members were provided with copies of my research questions, objectives and interview questions prior to agreeing to be interviewed and their names have been changed to pseudonyms to protect identities.

\section{Data Collection Processes}

\section{Interview Locations}

Elwood and Martin (2000) write that "Participants who are given a choice about where they will be interviewed may feel more empowered in their interaction with the researcher...[and] so that they may choose a place where they will feel comfortable to speak freely" (Elwood \& Martin, 2000, p. 656). My objective for the staff interviews was to collect information on the overall services each organization provided to newcomer youth. Therefore, in choosing an interview location I was less concerned about with offering staff a neutral environment and more concerned with interviewing staff in a space which they found convenient and comfortable. All three staff interviewed chose a location that accommodated their busy work and personal schedules over a location that accommodated confidentiality. Two of the staff 
insisted for their interview to take place at work in a private location, despite the fact their participation in the interview may become known by other coworkers thus reducing confidentiality (Elwood \& Martin, 2000). The third staff member was unable to meet with the interviewer in person and therefore opted for completing submitting written responses to the interview questions.

For youth participants, however, confidentiality of their participation was important since their responses were more opinion and experience based. Therefore, youth participants were provided the choice of interview locations at either the Ryerson University library or a public library close to where they lived (Elwood \& Martin, 2000). Many of the youth opted for public libraries close to where they lived as these environments were familiar to them, and this familiarity would reduce feelings of anxiety during the interview process (Ceglowski \& Makovsky, 2012). In the library, private rooms were used as interviews should be held in a private place away from people who could overhear the youths' responses (Alderson \& Morrow, 2004). Although a few of the young people wished to have their interview take place at the location of their youth programming, this request was denied to prevent the youth participant from confusing the position of the researcher with a youth program staff thus preventing the youth from freely discussing their experiences within the youth program (Ceglowski \& Makovsky, 2012; Greig et al., 2007).

\section{Interview Processes}

As stated earlier, program staff from the mainstream, multicultural and ethno-specific organizations participated in structured interviews to generate data regarding factual information on their organization and the services they provide to newcomer youth. At the beginning of the 
staff interview, the interviewee was asked to read and sign a consent form outlining the research and interview processes, rights of the research participant, and confidentiality processes as well as complete a short demographic questionnaire. The interviewee also had the option to choose whether or not to be audio-recorded for the duration of the interview. Although the questions were professional in nature, staff could choose to elaborate on questions by providing personal examples; however, many chose to answer the questions from a professional standpoint. Those staff members who participated in the interview were given a letter of appreciation.

Youth interviewed from the mainstream, multicultural and ethno-specific organizations participated in semi-structured interviews. Establishing a positive and safe emotional environment was essential for interviewing the youth participants as Alderson and Morrow (2004) warn of research processes which may intrude "into people's lives, and cause them great distress and embarrassment either during the project or afterwards" (Alderson \& Morrow, 2004, p. 36). To reduce emotional risks during the interview process, I created a friendly environment by providing snacks, and starting the interview with informal warm up discussions and my own immigration story (Ceglowski \& Makovsky, 2012; Greig et al., 2007; Valencia Vega, 2011). I found that sharing my own family's immigration story before the youth shared their own helped "build trust and enable the participant to open up" (Valencia Vega, 2011, p. 44).

Before the interview began, together the youth and I would read over the consent form which the youth (and their guardian if under 16 years of age) had signed. The purpose of the research, the importance of consent, the confidentiality of their interview responses and participation, their rights within the research process and their right to stop or leave the interview at any time were explained to them and they were provided an opportunity ask any questions they may have. All of the youth participants were given the choice to consent to or refuse to be 
tape-recorded. Two out of the nine youth participants declined being tape-recorded. Those who chose to be recorded were also provided the option to control the recording, again balancing the power between interviewer and interviewee (Alderson \& Morrow, 2004). In case youth would become upset from the interview, I prepared a handout with the contact information of a local youth counselling centre and help line in case it was needed afterwards (Alderson \& Morrow, 2004). At the end of the interview, youth were provided a letter of appreciation and paid a $\$ 15$ honorarium for their time.

\section{Data Analysis}

All audio recordings of staff and youth interviews were transcribed verbatim with the assistance of the Write and Cite Program. Handwritten transcriptions of some of the youth interviews were also typed. All interview transcriptions were saved upon a password protected USB so as to continue protecting participant's confidentiality throughout the data analysis process (Alderson \& Morrow, 2004). Each participant's interview was carefully read over several times to fully grasp each individual's unique story and experiences before entering into the coding process (Braun \& Clarke, 2006).

Following Braun and Clarke's (2006) guide for thematic analysis, all interview transcriptions were reviewed, coded and analyzed to determine themes. First, interviews were divided into three sections: positive experiences, negative experiences and recommendations based off of the three different focal points within the interviews. I then grouped all of the positive experience sections of the interviews and conducted analytic coding based off of descriptor words, feelings or experiences that identified positive experiences within the youth program (i.e 'fun', 'friendships'). Once a long list of codes were identified, I organized the 
codes and associated phrases or words into one list to review for similarities and begin to form sub-themes such as 'staff-youth relationships', and 'external causes of negative experiences'. At this point it was evident that the youth's negative experiences were also mentioned within the positive experience and recommendation sections of the interview, so I returned to code again for descriptor words and feelings associated to negative experiences across all sections of the interviews. After the second stage of coding, I had three pages of subthemes under the sections of positive experiences, negative experiences and recommendations. From this point I reviewed the list to identify relationships and outliers between the sub-themes which were used to create the larger themes such as 'meaningful relationships' and 'place of growth' that best capture the overall experiences youth disclosed (Braun \& Clarke, 2006). It was at this point that I realized many of the themes could be interpreted along deductive reasoning (Archer \& Berdahl, 2011), as characteristics of environments which are socially inclusive, and fulfilled Laidlaw's (2003) four cornerstones of social inclusion. 


\section{FINDINGS}

Findings within this study seek to answer two questions: first, how do first and second generation youth view their community based youth programming? And second, how do their experiences reflect social inclusion or exclusion? The findings of this research study are displayed in four sections. The first section provides an overview of the interview participant demographics and characteristics based on the demographic questionnaires. The second section illustrates the successes and challenges with immigration experienced by youth participants and their families as expressed through their immigration stories. The third section provides a summary of the mandates and services provided by each of the mainstream, multicultural and ethno-specific organizations involved in this research. The information presented in the third section was collected from the organizational websites, promotional material and through staff interviews. The final section will present the main thematic findings amassed from the staff and youth interviews divided into categories of positive experiences, negative experiences and comparisons between the three types of organizations.

\section{Participant Characteristics}

\section{Staff Participants}

A structured interview was conducted with one staff member each, from the mainstream, multicultural and ethno-specific organizations participating in this study. All of the staff participants directly worked with youth in their organizations. Staff participants completed a demographic information questionnaire which inquired about their age, gender, ethnicity, languages spoken and whether they were born in Canada. Two of the staff interviewed identified as female and one identified as male. Staff members represented a diverse group from different 
ethnic backgrounds, with two of the staff coming from immigrant backgrounds themselves and identifying their ability to speak multiple languages. Staff participants were also young, as two out of three identified as being less than 30 years of age.

\section{Youth Participants}

Nine semi-structured interviews were conducted with three youth from each participating mainstream, multicultural and ethno-specific organization. Three of the youth identified as second generation, and six identified as first generation. Youth participants also completed a demographic questionnaire which collected the participant's age, gender, ethnicity, immigration class, immigration status, year of family's immigration and languages spoken. The majority of the youth participants were male and between the ages of 14 and 20 years old. The participants represented a diverse range of Asian ethnic identities. The question of immigration class revealed that youth respondents were mostly unaware of their immigration class upon arrival. Five of the six youth who immigrated to Canada choose to respond "no answer" when asked their immigration class. Five youth are currently permanent residents, while the other four are Canadian citizens (three by birth). The majority of youth participants have lived in Canada for over three years whereas one youth was in Canada for less than a year and three youths' (second generation) families had been here for 11-20 years. These numbers reveal the long term need for immigrant focused youth programming that extends past the three year funding guideline for settlement focused funding. An overview of the youth demographic data is provided in Table 1.0. 
Table 1.0 Youth Demographic Information (Total= 9 participants)

\begin{tabular}{|l|l|}
\hline \multicolumn{1}{|c|}{ Gender } & \\
\hline Male Age Breakdown & 8 \\
\hline Female Number of Youth Participants \\
\hline \multicolumn{1}{|c|}{ Ethnicity } & 1 \\
\hline Age 14-15 & \\
\hline Age 16-17 & 5 \\
\hline Age 18-20 & 2 \\
\hline & 2 \\
\hline Middle Eastern & \\
\hline South Asian & 2 \\
\hline South East Asian & 4 \\
\hline Iranian & 1 \\
\hline Afghan & 1 \\
\hline & 1 \\
\hline Born in Canada & \\
\hline No answer & 3 \\
\hline Family Class & 5 \\
\hline & 1 \\
\hline Permanent Resident & \\
\hline Canadian Citizen (Born) & 5 \\
\hline Canadian Citizen (immigrated) & 3 \\
\hline \multicolumn{1}{|c|}{ Family's Length of Time in Canada } & 1 \\
\hline Less than 1 year & \\
\hline 1-3 years & 1 \\
\hline 3-10 years & 1 \\
\hline 11-20 years & 4 \\
\hline \multicolumn{1}{|c|}{ Languages Spoken Other than English } & 3 \\
\hline Persian & 2 \\
\hline Bengali & 2 \\
\hline Tamil & 1 \\
\hline Konkani & 1 \\
\hline Hindi & 1 \\
\hline French & 1 \\
\hline Dari & 3 \\
\hline Pashto & 1 \\
\hline Urdu & 2 \\
\hline $\begin{array}{l}\text { Number of youth speaking 2 or more } \\
\text { languages }\end{array}$ & 3 \\
\hline & \\
\hline
\end{tabular}


The duration of the youths' participation in each of the youth programs ranged between three weeks and seven years depending on the organization. The frequency of participation in each year also varied between organizations. Youth from the ethno-specific organization attended regular youth program activities once a week for approximately two hours, and more if they participated in the additional religious workshops or prepared for cultural shows. For youth at the ethno-specific organization, the start of the youth program activities coincided with the end of the school day and provided them a place to socialize right after school. Youth from the mainstream organization participated up to three days per week, usually on the weekend for a total of three to twelve hours per week. Youth participants from the multicultural organizations participated in a youth leadership program which operated for three weeks during the summer for a total of 24 hours.

What attracted youth to attend these particular organizations? Several of the youth participants were drawn to the youth programs at these three organizations because they lived close by. Others youth travelled to attend the programs because such programs were not offered in their neighbourhoods.

\section{Organizational Profiles}

To portray the range of programs and services available to first and second generation youth in Toronto, three community organizations volunteered to act as sites for youth and staff participant recruitment. Two of the participating organizations are located in the community of North York and the third is located in Regent Park. The organizational profiles below detail the breadth of services each type of organization provides to first and second generation youth 
clients. The organizations are not identified by name, to provide anonymity for the participants in this study.

\section{Mainstream Organization}

The participating mainstream organization is an established community organization that values its ability to work directly with community members from all walks of life (RK interview, 2014). With the establishment of the Don't Ask Don't Tell policy in Toronto, which advocates that access to city services not be determined based on immigration status, the mainstream organization put policy into practice by eliminating requirements for program participants to disclose information regarding their immigration status or ethnic identity (RK Interview, 2014). The youth programs they offer "are inclusive. Any youth age thirteen to twenty-nine can register and attend our programs regardless of gender, nationality, ability or ethnicity" (RK interview, 2014).

The mainstream organization targets all individuals and families living in their neighbourhood and as RK summarizes, "We address the needs of the youths in the community, not newcomer specific" (RK interview, 2014). Their current youth programming supports approximately two-hundred community youth after school and on weekends (RK interview, 2014) through any challenges they may face during adolescence. Services provided are recreational and academic in nature providing opportunities for personal, educational and career mentorship (RK Interview, 2014). Youth interview participants were recruited from the mainstream organization's Boys' Club [pseudonym] which provides a safe environment for teenage boys to participate in gender-specific programming with a recreational and skill development focus. However, youth participants spoke openly of their participation also in the 
after-school, weekend and tutoring programs as well as the impact the mainstream organization had on their families.

\section{Multicultural Organization}

The participating multicultural organization is "a newcomer organization that focuses on settlement in all realms" (Love interview, 2014). The multicultural organization provides newcomer families with "a lot of excellent resources and referrals to sources... on how they can settle, thrive, and lead" (Love interview, 2014). All of the programs and services are free for newcomer clients to access, as many of the newcomer families they work with arrive with little financial wealth (Love Interview, 2014). Participants within the multicultural organization are considered eligible for services if they have a Permanent Resident number; however the organization recognizes the need to be flexible with this requirement. Love explains:

the way you can differentiate a newcomer in [funder's] minds is by their status. So if you're a permanent resident, a convention refugee, I can work with you...My organization has been generous and flexible with me in allowing in Canadians, to a degree. We've negotiated terms on...the percentage that can be Canadian, and how many [Permanent Residents] and what is the cut off if I'm not working with enough of the demographic I'm hired to work with...based on the funder's request.

Youth interview participants from the multicultural organizations were recruited from the Youth Leadership Program [pseudonym]. The Youth Leadership Program targets newcomer youth aged fourteen to eighteen, however the age of participants is flexible since "some youth are put in lower grades than they were back home...that means [we] can sometimes have a 21 year old who's in high school that walks in here, and is...technically an adult...but they're in a high school" so they are allowed to participate in the program (Love interview, 2014). The Youth Leadership Program provides programming in throughout the year consisting of 
leadership workshops with a recreational focus that includes basketball, art and dance components (Love interview, 2014). A staff member explains that Youth Leadership Program is:

reaching out to kids who might not be drawn in through leadership...the way [the staff member] thought of it...is Mind, Body, Soul. Leadership is aiming towards the mind...dance and basketball is the body and the arts is reaching towards more the soul (Love interview, 2014).

\section{Ethno-specific Organization}

The participating ethno-specific organization provides newcomer services with a focus on a singular ethno-cultural group (Kay Interview, 2014). Their Youth Program [pseudonym] specifically targets youth aged fourteen to twenty-nine who "have come from a war torn country and have experienced war and trauma" (Kay Interview, 2014). The majority of youth participants are first generation youth, however, there are some second generation youth who also attend the programming (Kay interview, 2014).

The ethno-specific Youth Program provides youth with services focussing on settlement and integration such as mentorship, skill development workshops, conflict resolution, volunteer opportunities and recreational activities (Kay Interview, 2014). The youth program also provides activities which connect youth to their cultural, linguistic and religious community and heritage (Kay interview, 2014). In summary, the ethno-specific organization's "programs have been successful...and if it continues like this it makes it easier for new youth to integrate" (Kay interview, 2014).

\section{Young People's Immigration Stories}

To build rapport between the researcher and the youth participants, both began the interview by telling their personal immigration story or the story of their family's immigration. 
These stories represent a diverse range of experiences from life in the country of origin to settlement in Toronto. In the narratives below, pseudonyms are used.

Overwhelmingly, the youth described their family's journey to Toronto as a multidestination journey. For some, Canada was not the intended final destination as Leo, a first generation youth describes:

We first went to Bulgaria, my Dad used to work there...Europeans, they don't give the immigrants much stuff, so it's not that good. My parents thought Canada would be a good place for us to be educated.

For other youth, Canada was the preferred immigration destination for reasons of education, employment and overall perceived better opportunity. In his immigration story, James explained, "[W]e arrived here, uh, seeking a better life in fact, that's why we immigrate. A better life, a better education, in fact". In the case of Z's father:

He first came to America... he couldn't find a job or anything so one of [Z's] other uncles live in Canada...so then he told [Z's] Dad about how it's like good over in Canada and like, there's lots of [employment] opportunities for [Z's] Dad and stuff.

In addition, Bob, a second generation youth said, "My Mom told me in Sri Lanka life was easier and you knew what you would do in the future, where in Canada there are more open doors".

Not all youth, however, told stories of pull factors as reasons for immigrating to Canada. For some youth, it was the push factor of insecurity that resulted in their families immigrating to Canada. Leo explains:

Yeah, in Afghanistan, we had problems and stuff...and Afghanistan is not a good place to live in because of war and stuff so [my father] decided to live in Canada because it's safe.

Their reasons for migration were not the only information youth shared within their stories. The youth also disclosed their family's struggles with settlement. In particular, 
challenges in learning English were experienced by almost all the youth. As Narybati explains, "[A]t first, we came here, none of us could speak good English. We all had English classes in our home country but none of us could speak English here as well as the others". Lemar and Victoria both describe how different family members adapted to speaking English at different paces:

[W]e didn't know language actually. So umm, none of us, well my older brothers kind of did, but me and my little siblings, my parents didn't know how to speak English so that was one of the struggles we had, plus it was a new culture, changes and everything (Lemar).

[I]t was the language barriers. That was like the most difficult, but...We came here at a young age, so it was like easier for me to learn the language as for my sister...it was harder for her to understand the language, but it was easier for us because that was like our everyday language at school (Victoria).

Bruce Wayne arrived in Toronto at a very young age, yet he describes still struggling with the language barrier:

I remember sometimes we miscommunicate in like kindergarten. I would say something, people didn't know what I was saying, I had to clarify, use hand motions, point to that stuff and they would get the message. And...it was kind of a struggle with friends because you know language barrier... But then as I started to um get better at English.

Even though the second generation youth did not struggle with learning English, Bob describes being impacted by his mother's struggle to learn the language: "[B]ack then my parents didn't know English, by age 12 I still needed to help my Mom translate for doctor's appointments etc."

Language ability was not the only factor that the youth related to their experiences of inclusion or exclusion during settlement. They also described the impact neighbourhood coethnic concentration or diversity had on their feelings of inclusion or exclusion. Narybati shares: 
So at first...we were in a house in [one neighbourhood]... and like no one, almost no one there were Persians, so everyone were speaking English and it was so hard for us to understand whatever it was they're saying. And after 25 days, when we moved to our new house, many people around us are Persians...So we didn't have that much difficulty to speak and stuff like that.

Z, a Bengali youth, agrees that a co-ethnic neighbourhood composition was a more inclusive environment for his family, stating that living in a neighbourhood with "a lot of Bengalis in the area...felt like they fit in so now we're like living okay and it's all good".

Lastly, youth who were more established in Canada shared stories of success and adaptation. Bruce Wayne describes how his parents overcame their struggles to be successful in Canada:

[M]y Dad...his job was pretty good in India, but when he came here, and start from the bottom again...and uh, yeah he struggled with that but he's really hard working and he came back (laughs) I guess? Umm, he has a record in his company for becoming manager in like seven years, I think. And, my Mom was a nurse in India, and when she came back here she had to go to school again, because to get to the Canadian standard. And, yeah, she's a nurse now too.

Victoria summarizes how over the years, she has adapted to a Canadian way of life:

Well, first it was really different, like the school system, the language spoken, everything was different but as we went on, we got used to it and now it's just normal (laughs). Now, maybe if we go back home, the education system there might be different than what we are here, so it might be weird there, but we're used to it now.

\section{Experiences of First and Second Immigrant Youth in Mainstream, Multicultural and Ethno-specific Youth Programming}

In these young people's immigration stories, community organizations were represented in different ways. For youth from the ethno-specific organization, the role that the organization played in aiding in the youth's settlement was strongly emphasized. In comparison, second generation youth from the mainstream organization described the organization as a place they 
have grown up in, but less so connected to their family's immigration story. The youth of the multicultural organization had mixed connections to their organization, with youth who had just arrived praising the organization's support in their settlement, whereas more established youth emphasized the organization's impact on their overall integration and social inclusion within the community.

\section{Positive Experiences}

Overall, youth shared more positive experiences than negative experiences regarding their participation in community youth programming. Themes that materialized throughout the interviews focused on positive feelings; meaningful relationships; place of growth; and connecting with community. The sections below discuss each of these themes in more detail.

\section{Positive feelings of 'happiness', 'belonging' and 'pride'.}

Youth participants shared their positive experiences by telling stories, making comparisons between their youth program and others and sharing lists of activities they enjoyed participating in, within the different community organizations. Youth also expressed their positive experiences by describing the feelings they felt within the youth programs. Three feelings in particular were identified multiple times by the youth: happiness, belonging and pride.

Many youth participants described feeling happy attending their community organization and participating in youth programming. Within his youth program, Narybayti says, "Actually I feel kind of happy...coz I'm usually with my friends [at the youth program] and we do a lot of fun things together...it feels good". Youth also described the feeling of happiness using the word 'fun' as a descriptor of activities which stood out to them as positive or happy experiences. 
Victoria, for example, says, "like the Taste of Asia practices, we had a lot of fun during that and...when we have the BBQ, we go to the beach and it's pretty fun". Many youth felt happy in youth programming due to the relationships they cultivated there.

Positive relationships within the program were connected to feelings of belonging in the program similar to belonging to a family which confirm Thomas' (2012) findings that youth form family-like relationships within youth programming. Victoria explains this feeling by saying, "I feel--I feel at home...it's not like a youth program or something, I don't count it as that, I count it as like they're my family members like outside of home. And they're always there for me". Bob from the mainstream organization elaborates and describes the home-like environment he experienced within his youth program, "I like the program, they are always inviting. They are welcoming. When you get there they don't show hate, they want you to be there to have fun. It feels like home since I know everybody".

In addition to feeling belonging in their youth programming, youth participants shared feelings of pride in relation to their youth programs. Some of the youth felt proud to represent their community organizations and youth programs. During youth program outings, Bob explains, "We are being supervised, we have expectations and we are representing [the mainstream organization]. We have to be aware of what we are doing and be on our best behaviour". In having a personal connection to these organizations and youth programs, youth felt valued in their community and felt that through their association with the organization they were held to a higher standard.

For youth within the ethno-specific youth programming, pride was not only connected to representing a great organization but was presented as a result of the opportunities provided to 
youth participants to represent their culture. Victoria spoke highly about her experiences performing with the youth program:

it's basically music and it's dance and so it's all fun and we wear our clothes and we practice and then we go and we represent our culture and that makes us feel proud so it's good and I love that about this program.

Youth, especially from the ethno-specific organization were very proud in sharing that their youth program was a place in which one could learn about their culture and have the freedom to also express that culture with others.

\section{Meaningful relationships.}

In the interviews youth discussed three types of relationships which impacted their positive experiences within each of the organizations. The three types of meaningful relationships were: friendships, staff-youth relationships, and program-youth relationships.

\section{Friendships.}

Friendship was an important theme which emerged from all of the youth's interviews. All three youth programs were seen as locations in which youth developed lasting friendships and were able to socialize with youth from similar and different backgrounds. Friendship as a major outcome of participation within youth programs was often witnessed by program staff. Love recalls, "Friendship...it's like the main thing that [youth have] been talking about here, is aww, I've made new friends". For some youth, creating friendships with other youth who shared the same settlement experiences was valuable remedy for feelings of isolation. As Lemar describes:

the other good thing about the programs here was, I met a lot of people who were new like me, [and] they had to uh tell us... what was the positive and negative things they were facing here so I kinda was able to connect myself with their 
experiences, so it just, it used to give me the feeling oh, I'm not the only one who has immigrated here....So I kinda was...not feeling too lonely I guess.

The youth programs were also a place for youth to build friendships with youth possessing different life experiences. Youth from the ethno-specific organization found that through the youth program they were able to meet youth from different neighbourhoods and schools in Toronto. Lemar elaborates:

I made a lot of friends actually that I would not have been able to make if I didn't come to the programs here...this was the only place I think that connected me to people who came from other communities, let's say they weren't all of them from the same area so there would've been no way I could have met them. Soo, yeah, this program was one of the biggest help in that case, to be socialized. To be more open to people, yeah.

Youth found that the youth programs also created a unique environment for sharing their different life experiences and thoughts with others while having the freedom to do so without judgement. Bruce Wayne shares:

I feel like my ideas are accepted because everyone else's...they might have the same objective, but their way of thinking is different, their experiences are different. So whatever I say, people cannot be like Oh no! You're wrong! No, it's my experience...so you feel kind of accepted.

Another youth, James adds, "[We] talk about whatever we want...I can express myself with no fear".

Youth also provided a multitude of examples of the types of activities they participated in which led to the development of friendship. Activities which promoted teamwork skills, such as problem solving games, team sports and event performances were all examples provided by the youth. One of the youth, $\mathrm{Z}$, describes the connection between his youth program basketball workshops and the friendships he has developed: "we have our own teams, so it helps us like, 
bond better like we have this teammate chemistry it's good and we've become closer as friends, with all my friends and stuff'.

\section{Youth-staff relationships.}

Another meaningful relationship youth developed within the youth programs was the relationship between program staff and youth. The importance of this relationship became quickly evident in all nine youth interviews. For many youth, program staff provided advice and support on a variety of youth issues. As Lemar shares, "they (youth program staff) used to tell me whatever need I uh faced or any problems at school, I can always come here and they're the one who were helping me with that". Often staff members provided assistance on navigating the school system or with homework as Z explains:

Well, uh [the youth program] helped me in my education so like um, he (program staff) would tutor us in the subjects we're weak in and uh and say uh we have some projects that are happening in school? We'd bring them to [the mainstream organization] and we'd work on them with [a staff member] and he'll give us like, uh tips and stuff.

Youth felt as if program staff understood their needs and also recognized them as individuals as James describes, "[program coordinator]... she knows her student and how to get them involved to programming individually. Know, some of us want to be alone, some others they're hyperactive... She knows what to do with each person". Youth program leaders were also described by many youth as positive role models, often living the lessons they were sharing with the youth. Narybayti describes his program leader as having "the best leadership, the best leader that could be for this program". Bruce Wayne says that his program coordinator created a certain positive atmosphere in the program and that the, "positivity and like the confidence that she has, and like the positive attitude um it brings people together". 
When youth were lacking confidence, program staff became their biggest motivators and supporters. James who struggled with settlement in Canada described his program leader as, “someone, just shouting near my ear...okay, you're not a loser, you're just midway, you can stand up, it's not over, it's just the beginning! Yeah, she's something like that to me...Yeah, she inspires me a lot". Victoria adds that, "especially [the program leaders], they helped me a lot, whether it was school, it was personal, they helped a lot. And, I'm hoping to continue getting help from them".

Program staff members are integral to success of each youth program and many youth stated that one of their main reasons for attending the youth program was to spend time with their favourite staff. Bob explains that activities at the mainstream organization are more fun because of the high level of staff participation:

The good thing I like about [the mainstream organization], when we do activities, the staff are also involved. They will be playing with us, do basketball training or play ping pong. Some programs, staff are just there to supervise us and sometimes it is funner when the staff is involved.

Several youth valued program staff so much that they labelled them as family. In Victoria's experience, the program staff are, "like family members, I don't count them as my supervisors, they're more like family". She goes on to describe how the staff members take the time to support her through her personal life whenever she needs it:

if you come at any point of the day, any time, they're always here for you and they sit down with you whether they have work or not, they just sit down with you and they have their little talk and if you're sad and if you're bored and stuff, they entertain you and they talk to you about a lot of stuff and it's fun to be around...like I can talk personal with them, like I can even tell them about my boy stuff and like stuff, they wouldn't mind, they wouldn't judge and that's what I like about them.

\section{Program-youth relationship.}


The relationship between youth program and youth was described as one of lifelong membership. As Victoria says, "school programs, they were for a couple of weeks but this [youth program] is like lifelong (laughs)". Wazowski agrees that the youth program, "it's kinda a program that's going to help you grow. And uh, the program is going to be with you all through high school and all through middle school as well, right". Even, when youth aged out, they still remained in contact with staff, connected with opportunities and viewed the youth programs as places where they could continue to come back. Lemar shares:

With staff I still have contact with [them]...wherever, whatever kind of events there is, I always receive a message from [them] that oh, uhh they would like me to be there as well, so that's kinda of uh biggest help I guess, that was...if I didn't come to these program I don't think I would have that kind of bond with anyone, with any staff...because I know I make friends at school but once I graduate from school I get so into university stuff so it's kinda hard to be in contact but these programs are one of the reasons where I can always come back and sit with them and have fun.

A testament to the lifelong impact the community organizations have on participants is that some of the staff members were past participants of the youth programs. Victoria says that the ethno-specific organization is different than other programs because:

they're continuing, like if you see this guy, who just has a [workshop] right now, he's been here since he was like 12. Yeah, like I've been seeing a lot of people like right now, the people, they're like a teacher and they've been going to this program. So it's like, I see (emphasis) myself there. Like I can come back from ten years and I can be like yeah, I was here and I can give sessions to the kids... and be like...I was a youth there and give personal experiences.

All three levels of relationships support the ability of youth programming to establish a community within their organizational structure for youth participants. However, the youth programs also help youth connect further with the larger community at a neighbourhood or city level.

\section{Connecting with community.}


Whether youth settle into a community due to immigration or simply because their family moved to a new neighbourhood, connecting with a new community can be a challenging activity for first and second generation youth to accomplish. First and second generation youth face many barriers to social inclusion in society and it can be difficult to establish new social networks within their new environment (Hynie et al., 2012). Throughout the youth interviews, youth programs were described as acting as facilitator in the process of reconnecting youth to their community. Through the program activities, youth:

get to know their community and participate in their community...to become strong members of society. They usually get to know other communities in their surrounding and participating minimizes the barriers they face in Canadian society whether that's in school or other places and it minimizes marginalization and their isolation (Kay, program staff).

Lemar agrees with Kay by stating:

I'm so thankful... because I know if I didn't come to this program I would be only school and home, so I wouldn't have any chance of meeting new people and get to know the community and go out.

James, a recent arrival to Canada struggled deeply with loneliness and found that his participation in youth program activities helped him build connections with a community that provided him support to re-evaluate his situation. As James explains, "[The program] involving [me in] the society again. And, yeah, it shows me that it's just a new beginning....yeah. And immigration is not as hard as I thought". James explains that before the youth program, "I believe that there's no change, there's no progress...[and] for the first month, I just needed, wanted to get back home, that was all. But now, I don't feel like that. I'm feeling better".

The ways in which youth participants connected to community differed from program to program, however several youth described experiences in which they were given opportunities to 
give back to the larger community. For the ethno-specific organization, giving back was often through community events, as Victoria describes, "we had a huge event for everyone in the community, the youths in the community and it was for the Ramadan". Whereas, the mainstream organization often gave back through community volunteering, as $\mathrm{Z}$ explains:

usually we go out and we clean the community so we go out picking up litter and stuff and we usually have lots of charity occasions at [the mainstream organizations] so we'll help out and we talk with them, the community members, ask what they'd like in the community centre, what would be good for them and stuff.

$\mathrm{Z}$ added that his level of community involvement drastically increased due to his participation in the program: "I wouldn't be as involved in the community as much, like before I was in the program so once I came in...every week we would go do something in the community so now it's like, I'm used to it, yeah".

\section{Place of growth.}

Through the interview process, youth were able to reflect on their participation in youth program activities. For some youth, their participation was one week in duration and for others it was several years. Nevertheless, through reflection, youth began to share stories of their individual growth within the youth program. The growth witnessed can be categorized into four main subthemes: adaptation, employment and academic skill development, empathy and selfconfidence.

\section{Adaptation to Canadian life.}

For many first generation participants, youth programming represented a place that provided them with the education, tools and support to adapt to the ways of Canadian life. One program staff, Kay, describes the variety of settlement needs that are often addressed within 
youth programming, "getting their health card, counselling, providing interpretation, their PR [Permanent Residence] card, citizenship, travel documents. We also do skills development workshops, and they are based on the youths' needs and we do mentorship...and this has been successful". Youth programs act as a one-stop shop for first generation youth to learn what they need to survive in Canadian systems that are generally not friendly to newcomers. As Lemar describes, "Uh, the programs actually were really educative I kinda understand everything how things work here [in Canada]...Um things that we didn't know, through these programs we kind of learned how to deal with it". He goes on to share the information he received from his youth program when he first arrived in Canada, "[They tell] you information about how things work here, school, how to choose your courses, what type of courses you need. Coz the school is totally different here than where I have been, so that was a big help".

Adapting to a new society can be difficult for youth and sometime youth struggle and become victim to peer pressure and criminal behaviour. Youth programs try to prevent this by preparing youth for such challenges. In addition to helping Lemar navigate through the education system, his youth program also taught him:

about drugs. Like I know how uh when I think still there is in high school how they kinda use drugs and everything, so how to uh keep myself away from those stuff. Cuz obviously when you don't know the language they can, it's easy to get into that stuff.

For another youth James, the youth leadership program helped him push through his struggles to see a positive future in Canada. As James describes:

you see I was uh, taken apart after I...left home. And this program really helps me to rebuild myself. And in fact, I find different aspects of myself. I was in the middle of the dark tunnel and this program, in fact was a light there, I just see uh further in front of my eyes. 
Many first generation youth arrive with inadequate English language skills which can slow their rate of adaptation. First generation youth found that youth programs provided a supportive space for improving their English skills. Youth program staff members try:

to get [youth] comfortable with English...they're here but they're so shy to make a mistake, to sound stupid and have grammar issues...So really working on the fact that making mistakes is okay, you know, it's more about the practice and trying, and in the trying that's where we're learning (Love).

Narybayti found that his youth program provided the perfect environment to improve his English skills as he describes, "the program is nice because the activities are good and... it helps us and it actually improves English kind of because we are in the place where everyone speaks English...just a few speak their own language".

\section{Employment and academic focused skill development.}

In addition to experiencing growth in their ability to adapt to Canadian way of life, youth were able to develop skills which will help them achieve inclusion in higher education and improve their employability. All three youth programs offered youth skill workshops on leadership, employment and academic topics, and according to one staff member, Kay:

a lot [of youth] have taken advantage of these workshops, helping them to get into university or college... They also gain leadership skills which is good for them in long run so in the future they can take role of leadership in the community.

Some youth were able to identify precise skills they gained from participating in various activities. Bruce Wayne describes the leadership skills that he developed in the youth leadership program, he says that he enjoyed the "games...coz we had to communicate with each other and sometimes um, we had to like, work together and that built communication and team work skills, which is important in life". Other youth drew connections between the activities they participated in and their future ambitions as Bob summarizes, "I only go to... a youth program for boys. They 
help develop our abilities, they teach us valuable lessons like learning things we can use for our careers".

Not only were skill building workshops provided for the youth, but programs also offered volunteer and job opportunities. One of the young people, Wazowski described an upcoming potential internship in the works for youth participants, stating:

[The staff at the mainstream organization] gives us a lot of opportunities, there's job opportunities...I think that a lot of us are going to be getting internships...And uh, for me that's going to help a lot since I'm trying to go into business.

Providing youth with volunteer opportunities was also identified as the reason many of the youth initially signed up for their youth programs, as Toronto students are required to complete volunteer hours for school credit. As Lemar said, "I obviously needed the 40 hours volunteering so that was another big help for me to get it" and Leo confirms that he attended the youth program, "To get my volunteer hours and then I liked it". Whether the youth participated in a volunteer program, received employment and academic related mentorship or attended a skillsbased workshop, for many youth, their participation in the youth program helped them imagine an attainable future for themselves. One youth summarizes this feeling by saying, "I can see my goals are reachable...I can reach them. In fact better, or sooner then back home. I uh I feel free now" (James).

\section{Development of empathy.}

The third way in which youth grew through their participation in youth programming was in the development of empathy towards one another. Youth described youth programs as an environment in which youth are provided space to learn how to support other youth in their lives. Bruce Wayne shared how youth participants developed a support system for each other, "if 
someone is unhappy for like another reason like a personal problem or a home problem then the group would try and comfort that person". Victoria describes her youth program as a place where she can share and be listened to:

when we come and no matter who is in the room, we get all personal [and]...we talk about random stuff and like. So, I don't think there is any privacy or anything so we don't have anything to keep away from each other so we are all like a family member.

Youth also learned how to respect each other's opinions and become open to new ways of thinking. Bruce Wayne from the multicultural organizations says that he learned:

how to like, accept people's different ideas and their way of thinking coz like you can't be right all the time. Sometimes someone else has a better strategy or something else that works that might get your brain ticking and may help you.

Love finds these moments when youth free their opinions and judgements very moving, she recalls that:

sometimes...[the youth will] go into really authentic self. It's just a pure communication, they're not filtering, they drop the mask, they're real. In that moment, we're having a heart to heart...For me, I think that's very important to have that chance for them to feel safe enough to let that mask down.

The ability for youth to gain empathy when interacting with other youth created a safe space within the walls of the youth programs. This safe space is one in which youth felt free build their self-confidence and are open to explore their identities.

\section{Growth in self-confidence.}

Growth in self-confidence was expressed by the youth in several different ways. The first way was through achieving a task they had previously not thought possible. Wazowski 
shared that when he first joined his boys program, he was not athletic, but that participating in the program:

actually has helped a lot. One thing is like it helps to spend my time right, rather I'd be sitting at home on the computer and stuff...It's helped me um, athletically, cuz I was pretty um un-athletic before, now I actually have something to like, strive for.

For Lemar, participating in youth programing helped him gain self-confidence and become more social and interactive with other youth:

I still remember when I come [to the youth program] now, they tell me oh you used to be in grade nine and like, shy and I used to just sit in a corner but now I'm totally different. So these programs are one of the biggest reasons that uh made me that (different).

When youth look back at their participation in youth programming, they are able to recognize their growth and in evaluations conducted after the youth leadership program the number one stated outcome by youth was a growth in self-confidence. One staff confirms, "I've heard a lot of people being like, I'm more confident now, less afraid" (Love).

\section{Negative Experiences}

Hebert, Wilkinson \& Ali (2008) write that youth exhibit strong attachments to certain places in their lives, however, "[s]trong as these attachments are, they do not prevent the youth from noticing the ugly and unsafe aspects of places they are attached to" (p. 63). When questioned about their negative youth program experiences, some youth objected to the existence of negative experiences. One youth replied, “I don't have any bad experiences... When I don't feel good I don't come here, I only come when I feel good" (Leo). However, despite the insistence that there were no 'bad' experiences within the youth programs, the majority of the youth made critical comments about their youth programs, identified areas which needed 
improvement, and suggested solutions to the problems. Negative experiences disclosed during the interviews were divided into three themes: Causes of Negative Experiences, Experiences of Exclusion, and Challenges with Program Funding.

\section{Causes of negative experiences.}

Learning the various causes of negative experiences within the three youth programs was an interesting result of the interview process. Examining the origins of some of the negative experiences shared with me, I realised that some of the negative experiences originated within the youth program whereas others were a product of external environmental factors. Internal and external causes of negative experiences within youth programming will be discussed below.

\section{Internal causes of negative experiences.}

The majority of negative experiences occurring within the youth programs were a product of internal instigators. The primary instigators of issues within the youth programs were the youth participants themselves. In particular, youth participants' poor attendance, negative behaviour and interactions with other youth were directly linked to other youths' negative experiences within the programming. Victoria, for example, found that some activities had irregular youth attendance which affected her overall experience with the activity. Victoria explains:

sometimes the Taste of Asia program can get like annoying because not everyone shows up, you know... [coz] you know if not all participants like...if they don't show up how are you going to practice and stuff and how are you going to figure out things but we made it through and it was a good event so I can talk about that, either negative or positive, yeah.

In other cases, youth mentioned how some young people's behaviour can have consequences for all youth participants. Bob explains, "If all of us are not at our best behaviour sometimes they 
can cancel fieldtrips or special events for whoever that is not behaving. That's only the worst thing that happens".

The second cause of negative experiences was due to youth and program staff miscommunicating program objectives and activity expectations. For example, James found that many younger youth participants did not understand the purpose of the youth leadership program and therefore, "they are there to play games, to spend their times and to fill their volunteer hours. That's it." This contradicted James' perceived objective of the leadership program: participating in discussions on leadership topics. In the mainstream organization, youth mentioned that sometimes youth attend programming only for the sports and ignore the importance of participating in the employment and leadership workshops offered. As Bob summarizes, "most of the youth, we always want to have fun, sports, fieldtrips and stuff and when stuff like learning or when people come in and talk about anything besides that, some guys don't like that, they find it boring". $\mathrm{Z}$ adds that the program staff, "want us just to learn, and be like inspired like...like [the staff member] does these workshops to give us an idea of what we want to become when we grow up...but like, some of them are just boring". It is important for youth programming to provide educational and youth development focused programming, however, it appears that sometimes it is a challenge to get youth involved in this programming as they are more willing to participate in an activity that is fun and more recreational in nature.

\section{External causes of negative experiences.}

Sometimes causes of negative experiences within the youth program are due to issues occurring within youth program's external environment or neighbourhood. For youth attending 
the mainstream organizations, neighbourhood safety concerns sometimes interfered with programming. As Z explains:

Uh, so the neighbourhood, around [the mainstream organization]...it's a quiet neighbourhood but...it's kind of a bad neighbourhood... sometimes when we leave the gym open and we go to another room, one day we came back... and like our phones were stolen and stuff...so like, it was hard for us, and like...we used to go home, like in the winter time it'd be like night...so we'd be worried to go home because...it's really dark and stuff and a lot of gang stuff happens outside.

Based on my personal experiences, external causes are more difficult to address, however youth gave examples of program staff looking for creative solutions to these issues so that they can continue to run a safe program. One solution developed in response to safety issues pertaining to youth walking home in the dark was that staff began to walk the youth home after program. $\mathrm{Z}$ explains that his program staff would "walk us halfway through the community because we all live in, around the same area so it would be a safe walk with [him]".

\section{Experiences of exclusion.}

It was difficult to determine whether youth faced exclusion in the youth programs as the youth who participated regularly in programming did so mainly because they felt included there. What was determined through the stories of youth participants is that only some witnessed youth experience episodes of exclusion, with the result that they left the programs and usually did not return. The few youth who witnessed or experienced exclusion, described it in very different ways. For one youth, Z, exclusion was due to clashing personality traits or interests:

there were some kids that came in the program but they feel excluded and they, coz they feel like they didn't belong here. Coz a lot of people in our program, they're like rowdy and like party types of people but some of them that come are calm and they don't feel like they belong there because in some sports, they're not good at so they feel excluded because they're not good at that specific sport that we play a lot. 
This echoed in Bruce Wayne's case; he experienced exclusion due to his shy nature: "just the first couple of classes you don't feel like you know anybody...that feeling of like, isolation".

An additional dimension was presented by Lemar who experienced exclusion in his youth program because his experiences as a first generation youth were different than youth who were born in Canada or who had lived here longer. Lemar explains:

Um, the bad experiences were, there was people living here for longer time then I was uh so obviously I was kind of different than them, I didn't know the language so uh, it was hard for me to talk and also the lifestyle, mine was I believe different than them. Yeah, so they were totally different from what I was. So I was feeling that I'm not the same like in the same circle kind of thing. So I was just different.

\section{Challenges with program funding.}

While youth related their negative experiences to instances of exclusion and internal and external program interactions, program staff in their interviews described challenges with program funding which lead to negative experiences for youth program participants. Youth participants were unaware of the connection between program funding and potential negative experiences but this connection emerged from staff interviews.

Program staff members were thankful for the funding their programs receive but were ready to note that "More funding [would] increase [their] capabilities to offer more to the youths" (RK). While program funding ensures programs are available to youth, funding stipulations may limit the resources programs can use and control the manner in which programs are operated. Love's organization receives funding to provide bus tickets and snacks to their youth participants; however, the amount of funds received does not adequately address the existing need. Love explains: 
We get TTC (Toronto Transit Commission) which is awesome, I'm so thankful for it, it really provides accessibility...[however] they don't provide enough for everyone in the program. How do we differentiate between who gets TTC, who really needs it and which families don't? Also, you know, they do give a lot of funding for the materials and the food and things like that but adults eat differently than youth. And it's really hard to get them to realize that if I bring in granola bars, no matter how many I bring, it's not going to fill them up... So in some ways I don't take for granted the fact that I'm being offered funding at all...so it's a double edged sword in the fact that I am thankful to have funding at all, there's definitely some limitations in the funding and there's definitely some challenges in trying to present it fairly.

Kay, another staff member adds that reduced funding has resulted in certain experiences being removed from the youth programming:

There are certain programs or events, classes or field trips that due to the funding we can't do. Especially the funding cuts have made it less available. Most of the youth are from low-income families therefore it's hard for them to access some of the resources by themselves.

In sum, the youth programs were all affected by negative experiences in some fashion. However, the youth participants did not find these experiences overwhelming but instead addressed them as opportunities for change. After sharing their negative experiences, youth presented their ideas for solutions that could transform their negative experiences into positive experiences. These solutions will be discussed within the recommendations section of this study.

\section{Comparisons on Cultural Experiences of Youth in Mainstream, Multicultural and Ethno- specific Organizations}

In transcribing and coding the youth and staff interviews, differences emerged in the experiences of the youth among the mainstream, multicultural and ethno-specific organizations. In particular, differences in experiences were observed when comparing the ethno-specific organization to the other two organizations. The ethno-specific organization provided its youth clients with unique services that addressed their ethno-specific linguistic and cultural needs. As 
one staff, Kay reports youth choose to attend the ethno-specific organization over more multicultural and mainstream organizations because the ethno-specific organization "is very culturally sensitive as [the youth] won't be able to be as comfortable in other places that are not as culturally aware. Also there's a huge [co-ethnic] population around here and clients feel free and more comfortable".

The ethno-specific organization provided youth with flexibility in regards to language use within the youth program. Youth were able to speak English or their native language which accommodated those youth who were still learning English. Lemar explains his bilingual experience within the youth program, "Uh, [the program] was done both in my language and also English coz I obviously wanted to improve my English and then uh if I didn't know anything, like the words that I didn't understand, I was able to ask in my own language”.

Another unique feature of the ethno-specific organizations was the opportunity for youth to learn more about their culture through music, dance and religious workshops. Receiving support in celebrating their culture was extremely empowering for youth participants. Victoria summarizes the feelings of empowerment expressed by all three participants by saying, "we represent our culture and that makes us feel proud so it's good and I love that about this program".

All three youth from the ethno-specific organization enjoyed the youth program for providing an environment specific for their cultural group. However, Lemar also saw the value in having ethnic diversity within youth programs, in that within the ethno-specific organization:

I didn't have a lot of people from other communities in this program...through the other programs that I went there was people from different countries who speak the different language, culture was totally different so I kinda got to know their 
culture, about their lives... how is their experiences so it was fun to kinda share our experiences with them as well.

In the more ethnically diverse mainstream and multicultural organizations, mention of cultural programming was non-existent. Although these organizations appeared not to provide cultural programming for their participants, culture was central to some of the programs' challenges. One of the staff at the multicultural organization described the challenges associated with navigating multiple cultures within one very Western-valued leadership program. Youths' cultural values sometimes prevented them from participating fully in the activities, as Love explains:

[Some of the youth] have that perception like, I'm the teacher or I'm the authority...if I'm wrong, they can't correct me...[Canadian] communication is more relaxed and we don't have the same values placed on politeness...I never know what can come off as offensive to certain people, or personalities, or cultures or things like that so this is a constant learning of like...how do I communicate with all these different [youths]?...And this is a learning process for me and especially working in a multicultural organization.

In addition to difficulties with cross-cultural communication, culture was also seen to divide youth participants from the multicultural organization into groups based on ethnicity, preventing them from interacting with other youth participants. Bruce Wayne describes the cultural divisions within the multicultural youth program:

Well, two main groups are Filipino and Persian...And, both, I have never seen them together a lot. It will mostly be like Filipinos on one side of the room and Persian people on the other side of the room in little groups.

Fueling these divisions are the youths' need for inclusion and their need to speak their language within their ethnic group. As Love explains:

[The youth are] very comfortable with the language, the idiosyncrasies of people with their own culture. They get each other, they get their jokes, they're worried 
about their English so they're afraid to kind of branch out, are people going to judge them?

When asked how he felt about the cultural divisions in the youth leadership program, Bruce

Wayne, a youth who is neither Filipino nor Persian, stated about these two groups that:

I think uh I understand why [they do it]. But I also think they should be more outgoing and talk to like people of different backgrounds... They should explore more, they should talk to someone else in English, try to practice because they're not, wherever they are in life there aren't always going to be [people of the same ethnic background] there.

Very little regarding culture emerged from the mainstream organization's interview participants. One youth, Z, mentioned that it was easier to be friends with other youth from the same ethnic background:

And most of my friends are [co-ethnics] because I grew up with them. The friends you've seen at [the mainstream organization], I grew up with them because they're part of my like community and stuff and our parents know each other so it was easier for us to become friends.

In the mainstream organization's staff interview, the cultural makeup of the program was not discussed in order to follow the Don't Ask Don't Tell policy. Despite this, the mainstream organization insisted that the youth program was inclusive to youth of all nationality and ethnicity (RK). 


\section{DISCUSSION}

\section{Introduction to the Discussion}

The purpose of this study was to answer two questions: first, how do first and second generation youth view their community based youth programming? And second, how do their experiences reflect social inclusion or exclusion? The findings section provided the reader with the answer to the first question by presenting the range of positive and negative experiences youth experienced within the three participating multicultural, mainstream and ethno-specific organizations. Based on my interviews, it is evident that community youth programming provide overwhelmingly positive experiences to first and second generation youth, whether offered through mainstream, multicultural or ethno-specific organizations. Positive experiences were represented through positive feeling associated within the programs, opportunities for growth, the development of meaningful relationships and the program's ability to connect youth with their community. The community youth programs studied also have many opportunities for improvement to mediate negative experiences within their activities based off of the feedback received from program youth and staff. Negative experiences included internal and external disturbances between the youth program and their participants or neighbourhood, experiences of exclusion and the challenges associated with funding.

In this next section, will address the second research question by discussing how the youths' experiences reflect social inclusion or exclusion. Youths' experiences will be discussed focusing on each of Laidlaw's (2003) five cornerstones of social inclusion: Valued Recognition, Human Development, Involvement and Engagement, Proximity and Material Wellbeing. This section will also discuss comparisons between the findings presented in this study and Thomas' 
(2012) study which looks at the relationship between youth settlement services and social inclusion.

\section{Valued Recognition}

The first cornerstone to Laidlaw's framework of social inclusion is valued recognition which can be interpreted as looking at the opportunities for youth to both receive and give respect in each of the youth programs. Intertwined with this cornerstone are notions of recognition of differences (culture, gender, etc.). The findings relate to valued recognition in two main ways: in the development of positive relationships and youths' freedom of expression and cultural association.

\section{Positive relationships.}

Hebert and Alama (2008) write that "Friendship is key to the struggle for recognition, rights, solidarity, all parts of relational citizenship and social networks" (p. 94). In all three programs, developing friendships with youth and program staff was directly positively linked to how the youth felt about their overall experience within all three youth programs. Friendships with other youth in the program, made the youth program experience 'fun', 'happy' and created an environment in which youth felt they were part of a family - that they belonged. Friendships play an integral role in aiding youth to develop their confidence to build positive relationships which facilitate their social inclusion (Karlovic, 2004). Activities supported youth in developing empathy and respect for one another and youth supported each other whether in times of personal crisis or during teamwork on the basketball court.

Program staff members act as mentors, mirroring positive youth-adult relationships by listening to them and navigating the young people through various challenges in their lives. 
Youths' close relationships with program staff helped them in understanding themselves and their opportunities for the future, something that is challenging to figure out by youth experiencing social isolation (Zhou 1997). Youth described their leaders in positive terms such as 'family', 'supportive', 'there for me' and were quick to defend the good work that their program leaders were doing which reflects that youth recognized their leaders' value in the programs.

In his study on social inclusion and newcomer youth in multicultural programming, Thomas found that participation in settlement services increased youth's ability to create friendships, especially with individuals from diverse backgrounds, leading to greater involvement in Canadian society (Thomas, 2012). This was also true of the youth participating in this study. Through their participation in the youth programs, they were invited to socialize with other youth of different backgrounds, ages, and gender identities. The positive interactions youth had with their youth programs resulted in decreased feelings of isolation and increased involvement with programs outside of their community.

\section{Freedom of expression and cultural association.}

The concept of valued recognition was also evident in youths' responses surrounding experiences of freedom of expression and cultural association in their programs. Although not mentioned in the mainstream organization, youth from both the multicultural and ethno-specific organizations described activities which promoted the value of free expression, and provided youth with the opportunity to share their culture with others. Youth in the multicultural organization gained confidence in sharing their opinions on different leadership topics, comparing and contrasting their cultures through debates, and working on their cross-cultural 
communication in teamwork activities. Youth were acknowledged for their opinions which were validated, and they also learned how to respect others in times of differences of opinion.

Youth from the ethno-specific organization discussed freedom of cultural association as feeling that they belonged in a community with a strong ethnic and cultural identity and saw that they were supported by the program to pursue cultural teachings and publicly represent their culture in the larger community. Sometimes there is a fear that youth may become so reliant on their own ethnic group supports that they experience difficulty integrating into Canadian society (Ontario Council of Agencies Serving Immigrants, 2009). However, in this case, participation in the ethno-specific organization appeared to do the opposite for youth participants. In providing youth with the opportunity to voice their beliefs and celebrate their culture in a safe environment, they were provided with recognition and validation of their beliefs and culture, contributing to their sense of self. Like in Karlovic's (2004) research, youth from this organization felt included in the larger ethno-specific community outside of the organization, and they also had the confidence to interact with services and populations outside of their own community, thus increasing their social inclusion in the wider Canadian society.

\section{Human Development}

Laidlaw's (2003) second cornerstone of social inclusion is human development. Within the framework of social inclusion, human development is "[n]urturing the talents, skills, capacities and choices of [youth] to live a life they value and to make a contribution both they and others find worthwhile" (Omidvar \& Richmond, 2003, vii ). Do youth programs provide opportunities to youth to develop their abilities for achieving value in their life and contributing positively to others' lives? Youth and staff interviews confirmed that all three types of 
organizations were highly focused on human development as showcased through the activities they operated. The programs offered a plethora of skill, employment and education based workshops which assisted youth in challenging and overcoming systemic barriers to social inclusion and achieving success in their educational and career goals. Thomas' (2012) study also recognizes the ability of youth programming to offer first generation youth with skills training which directly improves youth's social inclusion.

Assistance with skill development and academic/employment mentorship are critical services for first and second generation youth as both groups currently face multiple barriers to achieving social inclusion in the Canadian education system and the job market (Karlovic, 2004, Ontario Council of Agencies Serving Immigrants, 2009; Quirke, 2011; Gonzalez, 2009; Hynie et al., 2012; Phan, 2004; Mesch et al., 2007, Ngo, 2009, Yan et al., 2008). Program staff members play an important role in providing assistance to youth with completing their homework, finessing their resumés and in navigating career and economic pathways to improve their ability to challenge economic and academic barriers to social inclusion in the larger community (Hebert \& Alama, 2008).

Thomas' (2012) study also finds that in addition to skills training, youth programs provide networking assistance for youth, providing opportunities that focus on preparing them for economic inclusion. Similar to Thomas' (2012) findings, youth in this study also described instances in which their program connected them to volunteering and internship opportunities in the wider community. In addition, youth found that fieldtrips to different neighbourhoods in Toronto allowed them to experience new activities and expand their realm of possibilities in regards to recreational, educational and employment options in their lives. 


\section{Involvement and Engagement}

Laidlaw's (2003) third cornerstone, Involvement and Engagement, focuses on opportunities for people to participate in community decisions that may directly or indirectly involve them. Unlike Thomas' (2012) study which showed that youth programs developed youths' ability for decision-making in their community (i.e. voting), the findings in this study did not reveal that youth were involved in this form of community decision making. However, youth in this study reported multiple opportunities to participate in the decision-making processes that occurred in the youth programs. Each program relied heavily on creating opportunities for feedback from the youth to direct their programming choices. In the mainstream organization, youth met regularly to decide the activities they would do together, whereas in the multicultural organization, the program leader made an effort to check in with the youth participants to confirm that they were engaged in and enjoying the activities.

However, this was also one of the areas that could be improved upon in each organization. Many of the negative experiences youth revealed were a result of miscommunication between youth and program staff. As well, in the findings, youth described witnessing instances of exclusion which were not really addressed by the youth programs and potentially led to those youth leaving the programming for good. Involvement and Engagement means more than listening to the voices of the regular youth participants, it also means searching out the minority, the youth who may feel excluded and making sure that they too are included in decision making in their youth program (Caidi \& Allard, 2005). 


\section{Proximity}

The fourth cornerstone of social inclusion is proximity which centres on "providing shared space to create positive interactions between people and decrease negative social distance between groups" (Omidvar \& Richmond, 2003, vii). Essentially, one of the main purposes behind providing youth programming is to create a safe environment for positive interactions. However, the stories by youth presented a mixture of successes and challenges in achieving this and each organization approached proximity in their programming differently.

\section{Proximity in the multicultural organization.}

The multicultural organization had similar results to Thomas' (2012) study of settlement services, in which youth reported that the youth program helped them to be more open-minded and develop relationships with youth from different ethnic groups and communities. At the same time, the multicultural organization struggled with youth forming exclusive groups made up of their own ethnic background in the program. Despite the multicultural organization's efforts for everyone to speak English in the youth program environment, which Thomas (2012) has linked to social inclusion, some youth found it difficult to build friendships with individuals from the two main ethnic groups as activities did not encourage proximity between the groups.

\section{Proximity in the mainstream organization.}

Within the mainstream organization, implementation of the Don't Ask Don't Tell policy created an environment within the program that was inclusive to neighbourhood youth of all ethnic or cultural backgrounds. However, youth from the mainstream organization reported no opportunities to connect with youth from different neighbourhoods in Toronto. 


\section{Proximity in the ethno-specific organization.}

The ethno-specific organization, unlike the other two organizations and Thomas' (2012) study, did not prevent youth from speaking their native language in program. Youth found that they were able to develop belonging and skills easier because they could use both their native language and English to communicate with other youth participants. The ethno-specific organization also facilitated opportunities for youth to interact with youth from other neighbourhoods, although the youth shared the same ethnic background. However, youth participants stated that participating in the ethnic-specific organization provided them with the tools and confidence to become involved in other youth programs and community activities in their spare time, thus connecting them to people of different backgrounds and assisting with their social inclusion into the more diverse Canadian society.

\section{Material Well-Being}

The last cornerstone of social inclusion is Material Well-Being or the ability of the organizations to foster youth's participation within the larger community through the provision of resources (Omidvar \& Richmond, 2003). Youth testimonials described several examples of material and non-material tools which assisted their social inclusion both within the program and in the larger community.

\section{Material tools.}

Food and transit fares were two material resources that youth described as breaking down barriers to social inclusion. Food satisfied a basic need of the youth, abating their hunger after school and allowing them to focus fully on skills development and socialization while participating in programs. The provision of transit fare bridged the barrier of economic 
exclusion, facilitating youths' access to the programs and to the many services provided to them in each organization.

\section{Non-material tools.}

Instead of material resources, Thomas' (2012) study focused on non-material resources such as assistance in the form of homework help and supporting youth to enter into postsecondary education. This was also true for all three participating organizations as well, focused on assisting youth in preparation for their academic and employment ambitions. Some of the resources provided were homework assistance, support with academic course selection, resumé building workshops, leadership workshops and volunteer and internship opportunities. Nonmaterial resources connected youth to skills that will be needed to overcome barriers currently faced by first and second generation youth in the Canadian education and employment systems.

In conclusion, this study demonstrates that community youth programming in several ways create spaces of social inclusion and through their efforts as reduce first and second generation youth's social exclusion within the larger society (Thomas, 2012). Societal exclusion is proactively addressed in three ways by community organizations in this stud. First, they attempt to provide equal opportunity for all youth to access their programming, often providing transit fares to youth who cannot afford transportation to the organization. Second, once youth are within the program, they provide skill development focused on helping the youth achieve inclusion and success within two of the most important societal structures: education and employment. Third, the community organizations proactively address issues of discrimination and isolation by creating a supportive community network of friends and mentors that continue 
with the youth even as they age out of the program, proving them with a place to belong that will exist their lifetime.

\section{Limitations of this Study}

There are several limitations to this research study which I would like to make note of. First, the small number of youth participants (nine in total), staff informants (three in total) and organizations (three in total) prevents me drawing any generalizations regarding first and second generation youth experiences within community youth programming. Any conclusions drawn from my research represents only the characteristics of the small sample group whom I interviewed on this topic. However, the study provides multiple useful new research strands that will hopefully be pursued in studies using larger samples.

Second, there is also a severe gender imbalance in this study regarding the representation of youth experiences as only one out of nine youth participants was female. This research study is thus lacking in the female voice of first and second generation youth experiences. Future studies will hopefully explore the differences and similarities among female and male youth participants in a youth programming environment; however with such a gender imbalance this study is unable to do so.

Third, I interviewed youth who were currently involved in the youth programming and who volunteered to provide information on their youth program. This may not have been the best sample to investigate negative experiences, as their voluntary participation in their youth programming may be an indicator of lack of negative experiences with this environment. To truly study negative experiences within the youth program environment, it would be worthwhile 
to include the voices of youth who no longer attend youth programs and ask them their reasons for leaving. 


\section{RECOMMENDATIONS}

The following recommendations section will suggest improvements to community organizations, funding bodies, and outline areas for further research. Some of the recommendations will be formed from questions which arose in my mind while analysing the findings. The majority of recommendations, however, are those I collected from the youth during the interview stage. When youth were asked to identify negative experiences in their youth programs, they were also asked if they had any recommendations for making improvements. It is important that this recommendations section also be used to showcase these young people's suggestions for changes.

\section{Recommendations for Community Organizations}

Some of the youths' negative experiences were a result of exclusive groups or cliques forming within the youth program. Bruce Wayne suggested matching youth from different backgrounds to work together on a task. He explains that this "would get them like, kind of away from their comfort zone but uh, yeah, it would just be like better because it's better than just hanging out with your own ethnicity all the time, you're trying something new". Youth also recommended for program staff to work with youth leaders in developing programming that showcases their talents to other youth and creates a mutual platform for creating relationships and sharing talents among youth in the programs.

Some of the youth participants identified a gap in age-appropriate programming for youth 18-21 years of age. First generation youth who arrive in Canada in this age range, often participate in high school classes even though they are legally considered adults. Since they are attending high school, they may be attending youth programs that normally cater to youth under 
age 18. Although older youth benefit greatly from the leadership and skill workshops provided in youth programming, it is also difficult for them to feel socially included in a program with youth several years younger. One of the youth participants, James, suggested that youth programs look at developing leadership programs for older youth who are between high-school and university.

Many youth recommendations for community organizations focused on adapting programming activities. Bruce Wayne who was in a leadership focused youth program, suggested that the program to incorporate more recreational programming. His reasoning for this change is that, "[m]ore recreational and athletic activities should be added to help youth develop friendships, fill time and lead healthier lives". Another youth, James, felt that activities should be taken to the wider community and be less confined to the youth program building. The youth explains that he would enjoy, "Maybe outside activities, in the society. You know, with other people, maybe? The kind of activities that we can have communication with real people in real lives".

One of the findings of this study focused on the crucial relationships youth had with program staff. Unfortunately, due to limited funding, many programs can only afford a limited number of paid staff. After recognizing the positive impact his program leaders had on the youth program, Wazowski said, "I think I would suggest, probably at least umm, one more staff, like in addition to the staff". He also suggested that programs look at expanding more opportunities for older youth to move into junior staff roles to support program staff. Provision of junior staff position for youth aging out of the program not only allows them to stay connected with the youth program and provides them with valuable work experience, but it would allow for the youth to give back to the program and share the skills and knowledge they have learned over the years with new youth participants. 
In response to incidents of exclusion within youth programming, youth had several suggestions to making activities and programming more inclusive for everyone. $\mathrm{Z}$ noticed that “when we usually play sports, it's always about who's good and who's not, but like if...there should, like when we play it should be that everyone wants to be like having fun and stuff'. His suggestion to include youth who may not be as athletic was to introduce new sports or games that no one was familiar with so that everyone would be experiencing that activity for the first time together without pressure to be good at it.

\section{Recommendations for Funding Bodies}

Community organizations rely on funding from public and private funding sources to operate their programs for first and second generation youth. This study presented the important role community organizations play in first and second generation youths' lives, creating a socially inclusive space in which youth can thrive. However, staff of the youth programs indicated that they are impacted by funding cuts, or that the funds provided do not address the expressed needs of clients. This results in some organizations having to choose which clients will receive or be cut off from resources.

I have several suggestions for funding bodies address these concerns. First, I suggest that funding organizations work closely with programs to develop sustainable funding agreements that focus on the needs of the youth. Resources such as food and transit fare which fulfill basic needs and allows youth to access services for those needs should be provided for all youth participants. Second, I suggest that funding bodies re-evaluate their measurements for success, working towards incorporating fewer statistics and more human feedback by interviewing participants of the program to determine if a program is successful. Ngo (2009) writes that youth 
serving agencies "need to extend their view of immigrant youth as merely recipients of services, and recognize their potential as contributors to the development of culturally responsive and youth-relevant services" (p. 95). I would like to see Ngo's (2009) recommendation extended to funding bodies so that they too are able to realize the potential of the youth who receive their funding.

Third, I ask that funding bodies consider the removal of client permanent residency as an eligibility criterion for community organizations to receive funding for 'newcomers'. As this study demonstrated, community organizations play an important role assisting first and second generation youth with their long-term social inclusion needs. The importance of settlement funding should not be negated, but settlement funding requirements should not act as a barrier to youth requiring assistance because these youth do not fit the funder's 'newcomer' definition (Omidvar \& Richmond, 2003). Omidvar and Richmond (2003) sate that "settlement funding and programming is focused on the initial stages of adaptation, in spite of the fact that the process of settlement continues throughout the life of the newcomer" (p. 8). Funding for first and second generation youth services should reflect this reality and provide sustainable funding to programs that provide services past the settlement stage toward full social inclusion.

\section{Recommendations for Further Research}

In analysing the findings for this study, two potential areas for further research became clear. First, while this study attempted to look at positive and negative experiences of youth, very few negative experiences, or instances of social exclusion were expressed. This is perplexing since similar environments like the school system have produced a lot of research on experiences of exclusion such as bullying and racism (Arthur, Chaves, Este, Frideres \& Hrycak, 
2008; Del Villar Nash, 2011; Graham \& Juvonen, 2002; Grossman \& Liang, 2008; Mesch, Turjeman \& Fishman, 2007; Phan, 2003). For community youth programs to produce almost no record of such events either means that these programs are doing something right that other youth-filled environments could learn from or it means that myself and Thomas (2012) were unable to include youth who had experienced exclusion within these settings in our interviews (see above for the limitations of my study). One young participant, Z, suggested that youth who have negative experiences no longer attend the programs. My recommendation for further research would be to explore this paradox in greater detail, perhaps trying to interview youth who have left a program. Simply increasing the interview participant numbers may also capture a greater range of experiences.

As noted in the limitations to the study, the second area for further research would be the female experience in community youth programming. I think this would be an interesting area of research to pursue because in my own work experiences in the community sector I have observed first generation female youth negotiate between their recreational activities and their family responsibilities and I did not hear about these experiences in the interviews with male youth participants. 


\section{CONCLUSION}

This study sought to explore the range of experiences of first and second generation youth within mainstream, multicultural and ethno-specific community youth programs. Two research questions were proposed: To explore the overall experiences of first and second generation youth within community youth programming and to see whether their experiences reflected social inclusion or exclusion. The literature review showed that little previous research has been conducted on this topic despite many first and second generation youth utilising these community services (Greenberg, 2013). Through interviews with nine youth and three program staff from three different community organizations in Toronto a clear representation of their overall positive and negative experiences within community youth programming in mainstream, multicultural and ethno-specific organizations emerged.

The findings section of this paper provided a better understanding of the variety of services first and second generation youth access through community youth programs. Services were recreational, educational and leadership focused in nature supporting youth with settlement issues all the way to addressing issues of social inclusion. Findings were also presented on the youth's positive and negative experiences in each of their youth programs. On the positive side, youth described feelings of happiness and belonging within their youth programs and were extremely proud to represent each organization as a participant. Through their involvement in youth programming, first and second generation youth developed meaningful relationships with other youth and program staff, and felt that their relationship to the programs themselves would be life-long. Youth programs supported them in many areas of growth which included helping youth learn to adapt to a Canadian way of life, providing them with skill development opportunities, helping youth learn empathy towards other youth participants and gaining self- 
confidence to pursue their dreams outside of the youth program. Lastly, through their participation within the different youth programs youth were able to connect at different levels with their local community.

This study revealed that youths' negative experiences were caused by both internal and external factors. Although few youth currently participating in the youth programs had experienced exclusion, several had witnessed it occurring to youth who may no longer come to youth programming. Lastly, program staff revealed that challenges with funding often result in negative programming experiences for youth participants.

In conclusion, using Laidlaw's (2003) cornerstones of social inclusion to analyse the study's findings revealed that all five cornerstones of social inclusion were evident within youth programs, making them places of social inclusion for first and second generation youth. This study has presented the reader with a wide range of positive and negative experiences occurring within community youth programming as well as many recommendations for change within programming, funding and future research. It has shone the light on the quality of services provided for first and second generation youth by community organizations as well as the challenges these organizations face in providing such services. This study has also tried to fully represent the youths' voices through the findings, discussion and recommendations sections. If we listen to the voices of first and second generation youth, we will have the ability to address their needs and change their futures. 


\section{APPENDICES}

\section{Appendix A: Interview Documents}

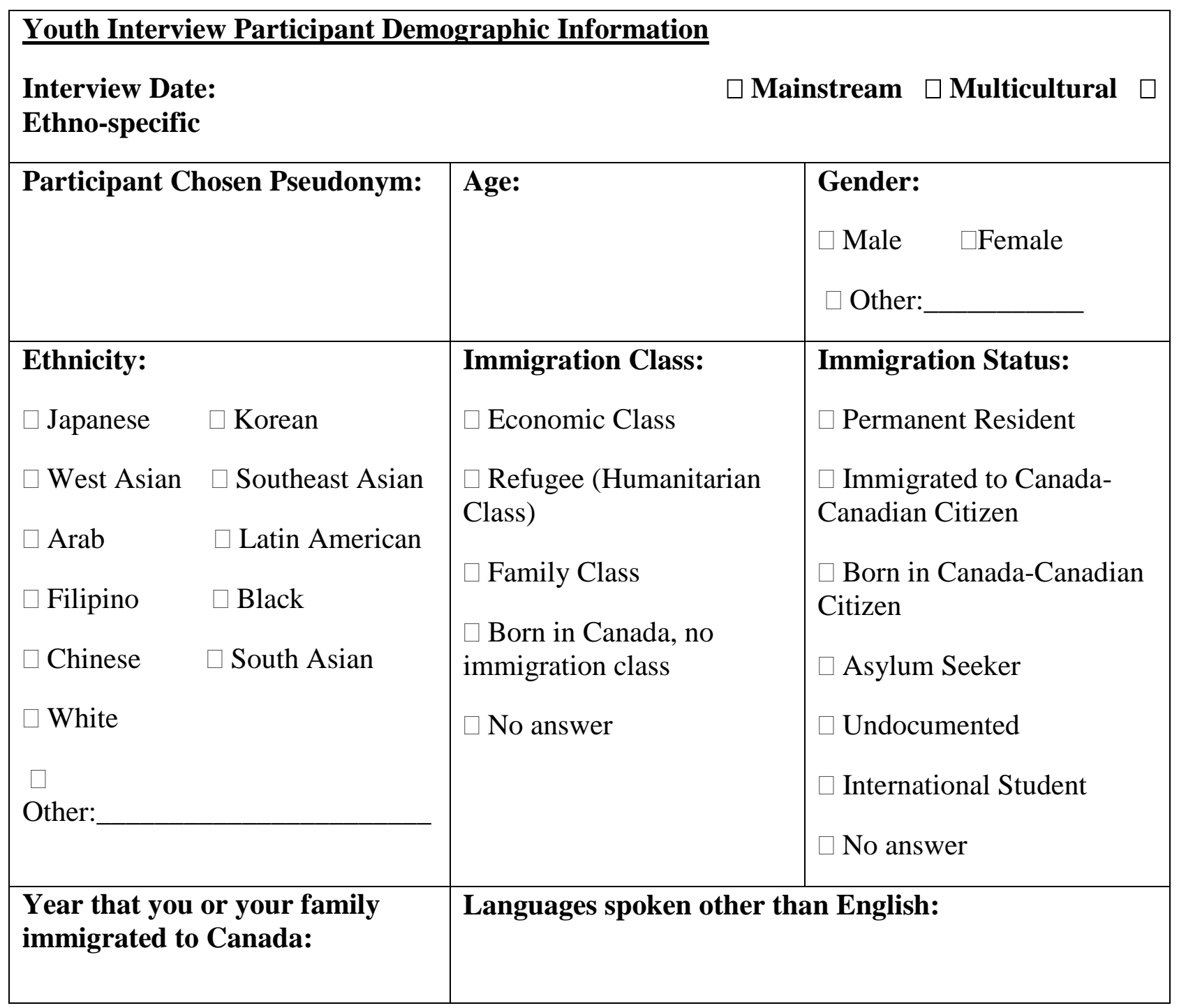




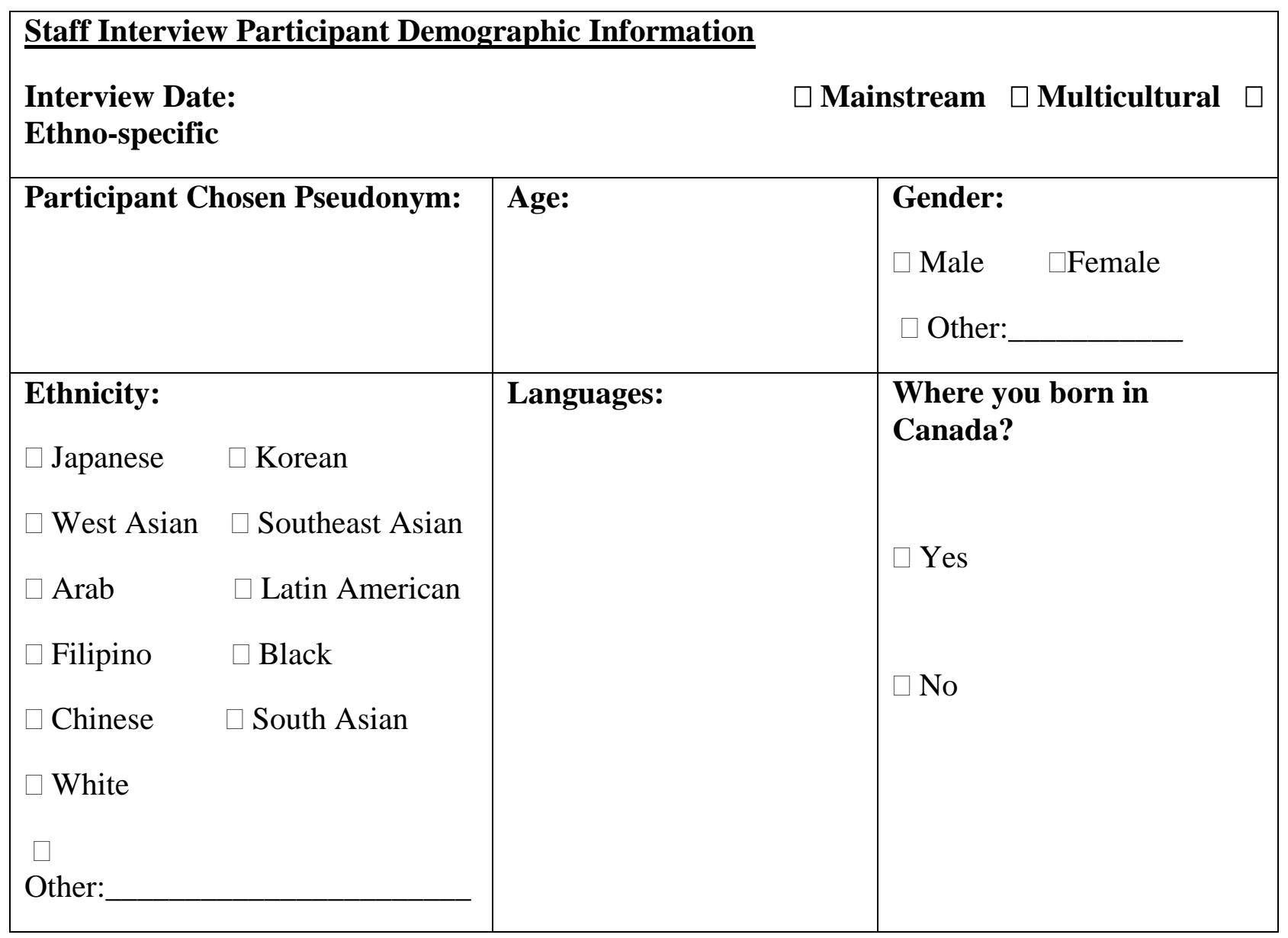




\section{RYERSON}

\section{UNIVERSITY}

\section{$\underline{\text { Staff Structured Interview }}$}

\section{Pre-Interview Checklist:}

Review purpose of study with the participant

Review and sign consent form

Participant completes personal information box and may choose a pseudonym.

Ask permission for interview to be recorded

Answer any questions participant may have regarding the interview process

\begin{tabular}{|l|l|l|}
\hline Interview Information & Location: & Type of Organization: \\
& & $\square$ Mainstream \\
Interview Date: & $\square$ Multicultural \\
& $\square$ Ethno-specific: \\
\hline Pseudonym of participant: & Time of Interview: \\
& \\
\hline
\end{tabular}

\section{Staff Interview Questions:}

1) Tell me about your organization?

2) What specific populations is your organization providing services for?

3) Tell me about the services your organization provides for newcomer youth.

4) What is the eligibility criterion for newcomer youth to attend this program?

5) How many youth attend your programs? How many participants are newcomer youth (meaning that they were born outside of Canada, including permanent residents, refugee claimants and new Canadian citizens)?

6) What ages are youth participants?

7) What ethnicities are youth participants?

8) What is the immigration status of your youth participants?

9) Why do newcomer youth attend your programs?

10) What positive experiences do youth have in your programs? What do they gain from the programs?

11) Are there any limitations or areas of improvement in your youth programs? 
12) What do you think are the benefits and limitations of providing services to newcomer youth within a mainstream, multicultural or ethno-specific environment?

13) Does this program address any needs specific to newcomer youth settlement or integration?

14) Is there anything else you would like to mention about your youth program or organization?

\section{Post Interview Checklist:}

Ask participant's permission to be contacted by telephone or email for a follow-up discussion to review their transcript or clarify points they made

$\square$ Ask participant if they would like a copy of the Major Research Paper (electronic or hard copy)

Present participant with thank-you letter 


\section{RYERSON}

\section{UNIVERSITY}

\section{Newcomer Youth Interview Guide}

\section{Pre-Interview Checklist:}

Review purpose of study with the participant

Review and sign consent form (ensure guardian consent form is signed)

Participant has complete demographic form and chosen a pseudonym.

If interview is to be recorded, ask participant if they would like to operate the tape recorder

Answer any questions participant may have regarding the interview process

\begin{tabular}{|l|l|l|}
\hline Interview Information & Location: & $\begin{array}{l}\text { Type of Organization: } \\
\square \text { Mainstream } \\
\text { Interview Date: }\end{array}$ \\
& $\square$ Multicultural \\
& $\square$ Ethno-specific \\
\hline Pseudonym of participant & Time of Interview: \\
\hline Honorarium Dispersed: & \\
$\square$ Yes & \\
$\square$ No-Reason: & \\
\hline
\end{tabular}

The following is a guideline of open-ended questions to ask the participants. As the conversation progresses, I will use probes to further explore the experiences of the participant:

Ice-breaker questions (used to build positive relationship with participant):

- Tell me about your/your family's immigration experience?

- What experiences stand out for you? Why?

\section{Program Details and Participation}


1. Tell me about the (insert name of youth program) you go to.

Prompts: - Why did you choose to go to this program?

-Did anyone connect you to this program?

-Are there special activities that attracted you?

2. How long have you been going to the program? How many times per week? For how many hours per day?

\section{Positive Experiences in Youth Programming}

3. What are some good memories/experiences of you participating in this program?

Prompts: -What activities are your favourite? Why?

-Has this program helped you in your life?

-Are there any good memories with staff or other youth from this program?

-What do you think other youth from the program would say are the best things about this program?

\section{Negative Experiences in Youth Programming}

4. What are some bad memories/experiences of you participating in this program?

Prompts: - What activities do you not like? Why?

-Have there been times during the program which you have felt unhappy?

-Are there any bad memories with staff or other youth from this program?

-What do you think other youth from the program would say are the worst things about this program?

\section{Participant Suggestions for Youth Programming}

5. Are there any things you would change with the youth program?

Prompts:-Do you have any ideas of how the program could better address your bad experience?

-Are there new activities, materials or equipment that you think is needed in the youth program?

Conclusion:

6. Is there anything else you would like to tell me about your participation in (insert program)?

\section{Post Interview Checklist:}


$\square$ Provide participant with counselling resources

$\square \quad$ Ask participant's permission to be contacted by telephone or email for a follow-up discussion to review their transcript or clarify points they made

$\square$ Ask participant if they would like a copy of the Major Research Paper

$\square$ Present participant with thank-you letter and honorarium 


\section{Appendix B: Recruitment Documents}

\section{RYERSON}

\section{Exploring Newcomer Youth Experiences within}

\section{Mainstream, Multicultural and Ethno-specific Youth Programming}

My name is Marleah Graff and I am finishing my graduate degree in Immigration and Settlement Studies at Ryerson University. My research study explores the positive and negative experiences of first and second generation newcomer youth within mainstream, multicultural and ethnospecific youth programming.

Very few research studies have looked at first and second generation newcomer youth and their experiences within youth programming happening after-school, evenings and weekends and facilitated by community programs. Little is also known about the services offered to first and second generation newcomer youth within the variety of mainstream, multicultural and ethnospecific types of programming and what newcomer youth participants like and dislike about these programs. "Exploring Newcomer Youth Experiences within Mainstream, Multicultural and Ethno-specific Youth Programming" is a study that gives first and second generation newcomer youth a chance to speak on their participation within youth programming and share their voices with the immigration and settlement programming and academic communities.

If you are interested in being interviewed for this study, please contact Marleah by email at

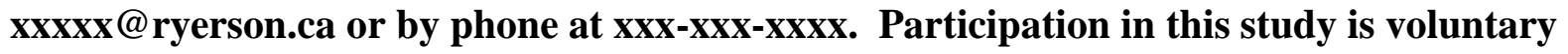
and confidential.

Youth participants must be between 12 and 18 years old, currently participate within (insert organization) youth programming and identify as a first or second generation newcomer. Youth who wish to participate must have consent of a legal guardian or parent. Interviews will last 4560 minutes and will take place at youth choice of the local Toronto Public Library or Ryerson University Library. Newcomer youth who are chosen for the study and attend their interview will receive a $\$ 15$ honorarium and a snack.

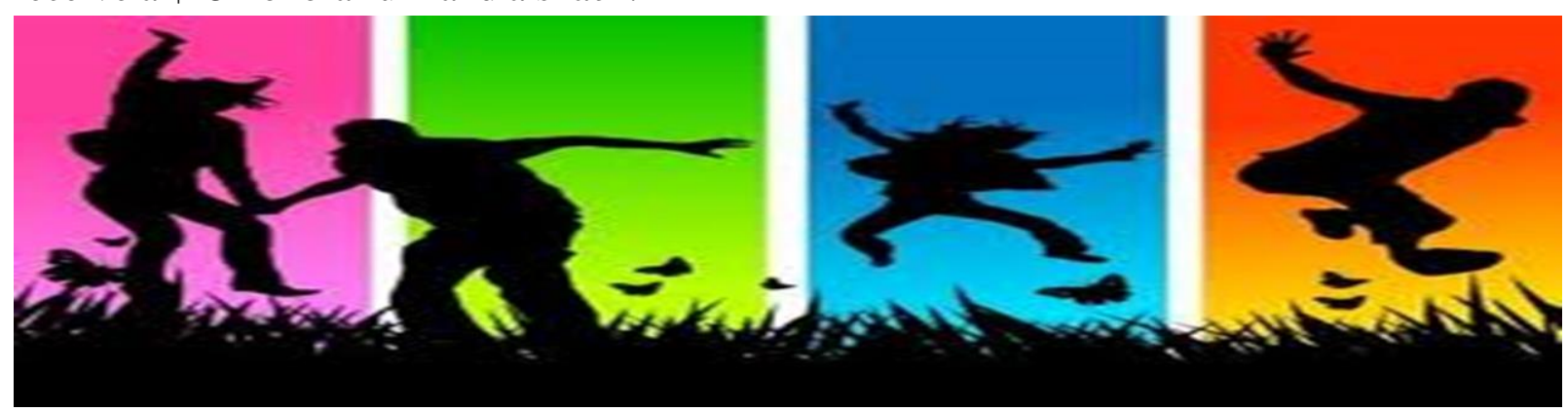




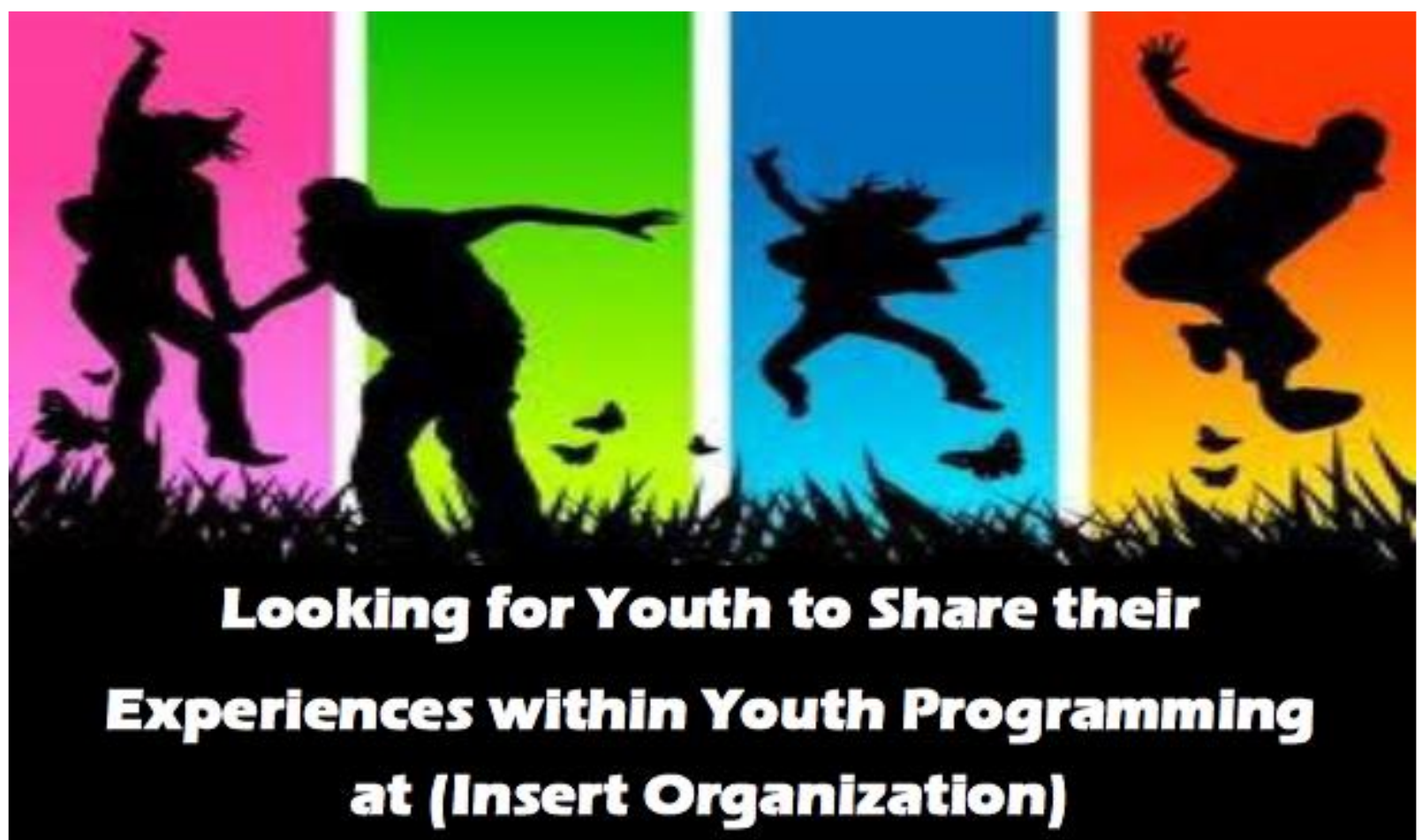

The "Exploring Newcomer Youth Experience within Mainstream, Multicultural and Ethno-specific Youth Programming" study is looking for first and second generation newcomer youth aged 12 to 18 who currently attend (insert organization) to be interviewed about their experiences within youth program activities. Newcomer youth interviewed will be paid $\$ 15.00$ for $45-60$ minute interview, with snacks included.

To keam more about the study and how you can participate, please attend the research recruitment presentation at (insert organization) on (insert date and time). Pizza will be provided for youth who attend the presentation.

If you are a first or second generation newcomer youth aged 12-18 and you are interested in being interviewed please contact Marleah Graff at 647-461-3872 or at mgraff@ryerson.ca

(Include your name, phone number or email and the program you attend) 


\section{REFERENCE LIST}

Alderson, P. \& Morrow, V. (2004). Ethics, social research and consulting with children and young people. Essex, England: Barnardo's.

Ali, M. (2008). Second generation youth in Toronto: Are we all multicultural? Canadian Diversity, 6(2), 87-89.

Archer, K. \& Berdahl, L. (2011). Explorations: Conducting empirical research in Canadian political science. Don Mills, Canada: Oxford University Press.

Arthur, N., Chaves, A., Este, D., Frideres, J. \& Hrycak, N. (2008). Perceived discrimination by children of immigrant parents: Responses and resiliency. Canadian Diversity, 6(2), 6974.

Batalova, J. \& Fix, M. (2011). Up for grabs: The gains and prospects of first-and-secondgeneration young adults. Retrieved from http://www.migrationpolicy.org/research /prospects-first-second-generation-young-adults-up-for-grabs

Beiser, M., Taa, B., Fenta-Wube, H., Pain, C. \& Araya, M. (2012). A comparison of levels and predictors of emotional problems among preadolescent Ethiopians in Addis Ababa, Ethiopia and Toronto, Canada. Transcultural Psychiatry, 49 (5), 651-677.

Braun, V. and Clarke, V. (2006). Using thematic analysis in psychology. Qualitative Research in Psychology, 3 (2), 77-101. doi: 10.1191/1478088706qp063oa

Caidi, N. \& Allard, D. (2005). Social inclusion of newcomers to Canada: An information problem? (CERIS Policy Matters Report 23). Retrieved from CERIS, the Ontario Metropolis Centre website: http://www.ceris.metropolis.net/wpcontent/uploads/pdf/research__publication/policy_matters/pm23.pdf

Ceglowski, D. \& Makovsky, T. (2012). Duoethnography with children. Ethnography and Education, 7(3), 283-295. doi: 10.1080/17457823.2012.717197

Colbert, J. A. (2012). The settlement of young newcomer children: Perspectives for policy and program development. In H. Bauder (Ed.), Immigration and settlement: Challenges, experiences, and opportunities (pp. 297-308). Toronto, ON: Canadian Scholars' Press.

Del Villar Nash, C. (2011). Bullying, ethnic discrimination, or both? A phenomenological study of the experiences of adolescent immigrants (Master's thesis). Retrieved from ProQuest database. (UMI No. MR85935)

Due, C., Riggs, D.W. \& Augoustinos, M. (2014). Research with children of migrant and refugee backgrounds: A review of child-centred research methods. Child Indicators Research 7 , 209-227. doi: 10.1007/s12187-013-9214-6

Duncan, H. (2003). Social inclusion, social capital, and immigration. Canadian Issues (April), Retrieved from ProQuest database: http://search.proquest.com.ezproxy.lib.ryerson. ca/docview/208699610?pq-origsite=summon 
Elwood, S. A. \& Martin, D.G. (2000). 'Placing' interviews: Location and scales of power in qualitative research. Professional Geographer 52(4), 649-657. doi: 10.1111/00330124.00253

Fine, M., Weis, L., Centrie, C. \& Roberts, R. (2000). Educating beyond the borders of schooling. Antropology \& Education Quarterly, 31(2), 131-151. doi: 10.2307/3196259

Fokkena, L. (2011). Moving beyond access: Class, race, gender, and technological literacy in afterschool programming. Radical Teacher, 90, 25-34. Retrieved from Academic OneFile database:http://go.galegroup.com.ezproxy.lib.ryerson.ca/ps/i.do?id=GALE\%7CA256930 $525 \& v=2.1 \& u=r p u \_m a i n \& i t=r \& p=A O N E \& s w=w \&$ authCount $=1$

Gonzalez, R. (2009). Beyond affirmation: How the school context facilitates racial/ethnic identity among Mexican American adolescents. Hispanic Journal of Behavioral Sciences, 31(1), 5-31. doi: 10.1177/0739986308328387

Graham, S. \& Juvonen, J. (2002). Ethnicity, peer harassment, and adjustment in middle school: An exploratory study. Journal of Early Adolescence, 22(2), 173-199. doi: $10.1177 / 0272431602022002003$

Greenberg, J. P. (2013). Determinants of after-school programming for school-age immigrant children. Children and Schools, 35(2), 101-111. doi: 10.1093/cs/cdt002

Greig, A., Taylor, J., \& MacKay, T. (2007). Doing research with children (2 ${ }^{\text {nd }}$ Ed.). London, England: Sage Publications.

Grossman, J. M. \& Liang, B. (2008). Discrimination distress among Chinese American adolescents. Journal of Youth and Adolescence, 37, 1-11. doi: 10.1007/s10964-007-92151

Guo, S., \& Guo, Y. (2012). Immigration, integration and welcoming communities: The role of ethnic community organizations. Edmonton, AB, CAN: Prairie Metropolis Centre. Retrieved from http://www.ebrary.com

Hebert, Y. \& Alama, E. (2008). Friendship as respect among second generation youth. Canadian Diversity, 6(2), 94-98.

Hebert, Y., Wilkinson, L. \& Ali, M. (2008). Six major characteristics of second generation youth in Toronto, Winnipeg, and Calgary. Canadian Diversity, 6(2), 63-68.

Herzog, P. S. (2011). Contextual inequalities in religious youth programming. Review of Religious Research, 53(2), 227-246. doi: 10.1007/s13644-011-0012-7

Hyman, I., Vu, N. \& Beiser, M. (2000). Post-migration stresses among southeast Asian refugee youth in Canada: A research note. Journal of Comparative Family Studies, 31(2), 281293.

Hynie, M., Guruge, S. \& Shakya, Y.B. (2012). Family relationships of Afghan, Karen and Sudanese refugee youth. Canadian Ethnic Studies, 44(3), 11-28. 
Karlovic, V. (2004). Living in Canada: Experiences of newcomer youth from the former Yugoslavia. (Master's thesis). Retrieved from ProQuest database. (UMI No. MR06995)

Khanlou, N. (2008). Psychosocial integration of second and third generation racialized youth in Canada. Canadian Diversity, 6(2), 54-57.

Kobayashi, A. (2008). A research and policy agenda for second generation Canadians: Introduction. Canadian Diversity, 6(2), 3-6.

Lim, A., Lo, L., \& Siemiatycki, M. (2005). Newcomer services in the Greater Toronto Area: An exploration of the range and funding sources of settlement services. Toronto, ON, CAN: CERIS - The Ontario Metropolis Centre. Retrieved from http://www.ebrary.com

Meinhard, A., \& Faridi, F. (2010). Diverse nonprofits for diverse communities: Organizational representation of visible minorities in Canada's four most diverse cities. Calgary, AB, CAN: Institute for Nonprofit Studies, Mount Royal College. Retrieved from http://www.ebrary.com

Mesch, G. S., Turjeman, H. \& Fishman, G. (2007). Perceived discrimination and the well-being of immigrant adolescents. Journal of Youth and Adolescence, 37, 592-604. doi: $10.1007 / \mathrm{s} 10964-007-9210-6$

Ngo, H. V. (2009). Patchwork, sidelining and marginalization: Services for immigrant youth. Journal of Immigrant and Refugee Studies, 7(1), 82-100. doi: $10.1080 / 15562940802687280$

Omidvar, R., \& Richmond, T. (2005). Immigrant settlement and social inclusion in Canada. Toronto, ON, CAN: The Ontario Metropolis Centre. Retrieved from http://maytree.com/PDF_Files/SummaryImmigrantSettlementAndSocialInclusion2003.pd $\mathrm{f}$

Ontario Council of Agencies Serving Immigrants. (2009). Newcomer youth settlement guide for service providers. Retrieved from Settlement.org website: http://atwork.settlement.org/ downloads/atwork/OCASI_SYNC_Youth_Guide_English.pdf Toronto: OCASI, 2009.

Phan, T. (2003). Life in school: Narratives of resiliency among Vietnamese-Canadian youth. Adolescence, 38(151), 555-566.

Phinney, J.S., Cantu, C.L. \& Kurtz, D.A. (1997). Ethnic and American identity as predictors of self-esteem among African, American, Latino and White adolescents. Journal of Youth and Adolescence, 26(2), 165-185.

Pratt, G. (2003/2004). Between homes: Displacement and belonging for second-generation Filipino-Canadian youths. BC Studies, 140, 41-68.

Qin, D. B., Way, N. \& Mukherjee, P. (2008). The other side of the model minority story: The familial and peer challenges faced by Chinese American adolescents. Youth and Society, 39(4), 480-506. doi: 10.1177/0044118X08314233 
Quirke, L. (2011). Exploring the settlement experiences and information practices of Afghan newcomer youth in Toronto. Canadian Journal of Information and Library Science, 35(4), 345-353.

Rossiter, M. J. \& Rossiter, K.R. (2009). Diamonds in the rough: Bridging gaps in supports for atrisk immigrant and refugee youth. International Migration and Integration, 10, 409-429. doi: 10.1007/s12134-009-0110-3

Sadiq, K. D. (2004). The two-tier settlement system: A review of current newcomer settlement services in Canada. Toronto, ON: CERIS - The Ontario Metropolis Centre. Retrieved from http://www.ebrary.com

Saloojee, A. (2005). Social inclusion, anti-racism and democratic citizenship. Toronto, ON: CERIS - The Ontario Metropolis Centre. Retrieved from http://www.ebrary.com

Sampson, R. \& Gifford, S.M. (2010). Place-making, settlement and well-being: The therapeutic landscapes of recently arrived youth with refugee backgrounds. Health and Place, 16, 116-131. doi:10.1016/j.healthplace.2009.09.004

Siahaan, F., Lee, D.Y. \& Kalist, D.E. (2014). Educational attainment of children of immigrants: Evidence from the national longitudinal survey of youth. Economics of Education Review, 38, 1-8. doi:10.1016/j.econedurev.2013.10.001

Stebbins, R. A. (2001). Exploratory research in the social sciences. doi: http://dx.doi.org/10.4135/9781412984249

Temple, B. \& Edwards, R. (2006). Limited exchanges: Approaches to involving people who do not speak English in research and service development. In B. Temple \& R. Moran (Eds.), Doing research with refugees: Issues and guidelines (chapt. 3). doi:

10.1332/policypress/9781861345981.001.0001

Tirone, S. \& Pedlar, A. (2005). Leisure, place, and diversity: The experiences of ethnic minority youth. Canadian Ethnic Studies, 37(2), 32-48.

Tinkler, T. J. (2006). Transitory freedom: Political discourses of refugee youth in a photography-based after-school program (Doctoral dissertation). Retrieved from ProQuest database. (3206425)

Thomas, C. (2012). Newcomers and social inclusion in Peel Region, Ontario: Examining the importance of settlement services. (Master's thesis). Retrieve from ProQuest database. (UMI No. MR93054)

Tyyskä, V. (2001). Long and winding road: Adolescents and youth in Canada today. Toronto, Canada: Canadian Scholars' Press.

Valencia Vega, M. C. (2011). 'Yo cuento': Latin American immigrant children tell their stories. (Master's thesis). Retrieved from http://issuu.com/investigadoreslatinoamericanos/ docs/newcomer_children_research_paper 
Valentine, G. \& Sporton, D. (2009). 'How other people see you, it's like nothing that's inside': The impact of the process of disidentification and disavowel on young people's subjectivities. Sociology, 43(4), 735-751.

Vo-Jutabha, E. D., Dinh, K., McHale, J. \& Valsiner, J. (2009). A qualitative analysis of Vietnamese adolescent identity exploration within and outside an ethnic enclave. Journal of Youth and Adolescence, 38, 672-690. doi: 10.1007/s10964-008-9365-9

Wu, Z., Schimmele, C. M., \& Hou, F. (2012). Co-ethnic concentration and trust in neighbourhoods. Vancouver, BC: Metropolis British Columbia. Retrieved from http://www.ebrary.com

Yan, M. C., Lauer, S. \& Jhangiani, S. (2008). Preliminary understanding of challenges in entering the job market: Experiences of new generation youth from visible minority immigrant families. Canadian Diversity, 6(2), 118-120.

Zhou, M. (1997). Growing up American: The challenge confronting immigrant children and children of immigrants. Annual Review of Sociology, 23, 63-95. 